\title{
The Galactic Habitable Zone I. Galactic Chemical Evolution
}

\author{
Guillermo Gonzalez, Donald Brownlee \\ Astronomy Department, University of Washington, P.O. Box 351580, Seattle, WA 98195 \\ USA \\ Peter Ward \\ Department of Geological Sciences, University of Washington, P.O. Box 351310, Seattle, \\ WA 98195 USA
}

Received _ ; accepted

accepted by Icarus 


\begin{abstract}
We propose the concept of a "Galactic Habitable Zone" (GHZ). Analogous to the Circumstellar Habitable Zone (CHZ), the GHZ is that region in the Milky Way where an Earth-like planet can retain liquid water on its surface and provide a long-term habitat for animal-like aerobic life. In this paper we examine the dependence of the GHZ on Galactic chemical evolution. The single most important factor is likely the dependence of terrestrial planet mass on the metallicity of its birth cloud. We estimate, very approximately, that a metallicity at least half that of the Sun is required to build a habitable terrestrial planet. The mass of a terrestrial planet has important consequences for interior heat loss, volatile inventory, and loss of atmosphere. A key issue is the production of planets that sustain plate tectonics, a critical recycling process that provides feedback to stabilize atmospheric temperatures on planets with oceans and atmospheres. Due to the more recent decline from the early intense star formation activity in the Milky Way, the concentration in the interstellar medium of the geophysically important radioisotopes, ${ }^{40} \mathrm{~K},{ }^{235,238} \mathrm{U},{ }^{232} \mathrm{Th}$, has been declining relative to Fe, an abundant element in the Earth. Also likely important are the relative abundances of $\mathrm{Si}$ and $\mathrm{Mg}$ to Fe, which affects the mass of the core relative to the mantle in a terrestrial planet. All these elements and isotopes vary with time and location in the Milky Way; thus, planetary systems forming in other locations and times in the Milky Way with the same metallicity as the Sun will not necessarily form habitable Earth-like planets. As a result of the radial Galactic metallicity gradient, the outer limit of the GHZ is set primarily by the minimum required metallicity to build large terrestrial planets. Regions of the Milky Way least likely to contain Earth-mass planets are the halo (including globular clusters), the thick disk, and the outer thin
\end{abstract}


disk. The bulge should contain Earth-mass planets, but stars in it have a mix of elements different from the Sun's. The existence of a luminosity-metallicity correlation among galaxies of all types means that many galaxies are too metal-poor to contain Earth-mass planets. Based on the observed luminosity function of nearby galaxies in the visual passband, we estimate that: 1) the Milky Way is among the $1.3 \%$ most luminous (and hence most metal-rich) galaxies, and 2) about $23 \%$ of stars in a typical ensemble of galaxies are more metal-rich than the average star in the Milky Way. The GHZ zone concept can be easily extrapolated to the universe as a whole, especially with regard to the changing star formation rate and its effect on metallicity and abundances of the long-lived radioisotopes.

Subject headings: Terrestrial Planets, Extrasolar Planets, Cosmochemistry, Planetary Formation 


\section{INTRODUCTION}

While its definition has varied somewhat over the last four decades, the Circumstellar Habitable Zone (CHZ) has generally been defined to be that region around a star where liquid water can exist on the surface of a terrestrial (i.e., Earth-like) planet for an extended period of time (Huang 1959; Shklovsky and Sagan 1966; Hart 1979). Estimates of its size have varied as climate models have been refined (Kasting et al. 1993; Franck et al. 2000b), but the present models do have some shortcomings. The most serious one is the adoption of the present Earth as the standard when applying the CHZ to other locations in the universe or other epochs in Earth's history. For example, the width of the CHZ is influenced by chemical weathering, but its efficiency is greatly increased by the presence of vascular plants (see Moulton and Berner 1998). Therefore, application of extant CHZ models to other planetary systems assumes that advanced plant life is already present. This inherent inconsistency notwithstanding, the CHZ is still a useful concept around which the presence of advanced life in the universe can be considered in a quantitative way.

Here we propose that, in an analogous fashion, there exists within galaxies a region favorable to the development and long-term maintenance of complex life comparable to terrestrial animals and complex plants. In the Milky Way this region forms an annulus in the disk, the boundaries of which are set by several Galactic-scale astrophysical processes that are likely to relate to habitability on terrestrial planets. Its location is variable with time, due, for example, to the evolution of the abundances of heavy elements (including the long-lived radioactive isotopes) in the interstellar medium. The inner limit of this newly defined Galactic Habitable Zone (GHZ), is set by high energy events such as supernova and gamma ray bursts, higher metallicity leading to orbital decay of planets, and gravitational perturbations of Oort cloud comets sufficient to cause frequent comet impacts (to be discussed in a future paper). These events cause mass extinctions and perturb complex life. 
The outer limit of the GHZ, the subject of this paper, is set by Galactic chemical evolution, and, in particular, the radial disk metallicity gradient. A certain minimum abundance of heavy elements is needed to fully assemble Earth-size planets in the CHZ. In addition, we argue that certain elements must be present with the right mix. The boundaries of the GHZ are not rigid, well definable limits, but are rather probabilistically defined.

Ours is not the first discussion of Galactic-scale constraints on habitability. Trimble (1997a,b) considered, in a general way, the composition of the Sun in the broader context of Galactic chemical evolution and how heavy element abundances in the interstellar medium constrains the timing and location of habitable planets. Clarke (1981) discussed the possible limitations on the habitability of a galaxy undergoing a Seyfert-like outburst. Several astronomers have been arguing since the early 1980's (e.g., Balazs 1988; Marochnik 1984) that the placement of the Sun's galactic orbit very near the corotation circle is an important requirement for habitability. Finally, many papers have been published since the early 1970's about the possible damaging effects of a nearby supernova (e.g., Brakenridge 1981; Ellis and Schramm, 1995). Each of these previous studies focused on only one type of Galactic-scale constraint. Tucker (1981) was one of the few to consider habitability in the Galaxy within a broader framework, but his treatment was very superficial and is now seriously outdated. Much of our motivation for investigating the possible link between Galactic-scale astrophysical processes and life on a terrestrial planet derives from the apparently anomalous values of several of the Sun's parameters, which Gonzalez (1999a,b) has interpreted within the framework of the Weak Anthropic Principle. For example, it seems an odd coincidence that we should happen to be living around a star with a space velocity relative to the Local Standard of Rest $\left(\nu_{\mathrm{LSR}}\right)$ smaller than most other nearby stars. The oddity of this situation can be removed if it can be shown that habitability favors a small value of $\nu_{\mathrm{LSR}}$. While this point was already addressed by Gonzalez (1999a,b), our present task is to bring the discussion within the framework of the GHZ. Our motivation 
also derives from much recent research showing that mass extinctions on Earth have severely affected the course of biotic evolution, and seemingly pose a threat to the survival of complex life on the surface of any planet (Ward and Brownlee 2000).

Our purpose herein is to present a unified, though not necessarily complete, treatment of the GHZ, including astrophysical processes over a wide range of space and time scales. In this first paper in a series we will address the constraints imposed on the GHZ by Galactic chemical evolution, focusing on the production history of biologically important elements. In future papers we will discuss threats from transient radiation events and asteroid and comet impacts and give quantitative estimates of the size and time evolution of the GHZ in the Milky Way and in other galaxies. In the following we begin with a presentation of our working definition of a habitable planet and a discussion of the basic geophysical requirements, followed by a discussion of the astrophysical processes that provide the basic planetary building blocks, compare the Milky Way habitability to other nearby galaxies, and end with a summary of the GHZ concept.

\section{DEFINING A HABITABLE PLANET}

Throughout the following we adopt the Earth as the reference habitable terrestrial planet, which we do for two simple reasons: 1) it is the only example we have, and 2) comparative planetology in the Solar System indicates that the Earth's habitability may be near optimal (especially for complex life). However, this second assumption may not be true for every parameter; for instance, the Earth may not be not have an optimal impact rate (impacts can have both positive and negative consequences). We employ the term habitability to refer to environmental requirements suitable for Earth-like animal-like aerobic life, not the broader range of conditions that might support microbial life. We have identified three necessary (though not sufficient) requirements for such a habitable 
terrestrial planet: 1) an ocean and some dry land, 2) moderately high $\mathrm{O}_{2}$ abundance, and 3) long-term climate stability. The moderately high $\mathrm{O}_{2}$ (and low $\mathrm{CO}_{2}$ ) abundance is a necessity for large mobile life on physiological grounds and also for building an ozone shield (McKay 1996). An ocean is required primarily for temperature regulation via the operation of a water cycle on a global scale, plate tectonics, and chemical weathering (on land). A completely ocean covered planet is excluded from consideration, because it eliminates solid surface-atmosphere interactions and limits the diversity of possible life (life on such a "waterworld" would likely be limited to that typical of deep sea thermal vents on the present Earth, if even that much). Long-term climate stability brings in many astrophysical and geophysical constraints, such as: stellar evolution, comet and asteroid impact rate, the presence of a large natural satellite, and a long-term planetary heat source to drive plate tectonics. In the present study we address those steps in the formation of such a habitable planet that are linked to the broader topic of Galactic chemical evolution. Implicit in our study is the assumption that deviations from Earth-like parameters lead to less habitable conditions. Full verification of this assumption may be possible in the future as models integrating planetary dynamics, geophysics, climate, and biology are further refined.

As an aside, we note that a large natural satellite orbiting a gas giant planet has been suggested as a possible alternative habitat to an Earth-like planet (e.g., Williams et al. 1997). However, such an environment is very likely to be less habitable for the following reasons: 1) comet collisions will be more frequent due to the strong gravity of the gas giant host, especially via captures into temporary orbits (also more frequent comet breakups will contribute to an increased collision probability), 2) the particle radiation levels are higher in the vicinity of a gas giant with a strong magnetic field like Jupiter (but some gas giants, like Saturn, have weaker fields), and 3) spin-orbit tidal locking will occur on a relatively short timescale. The first point was illustrated by the capture and subsequent breakup of comet Shoemaker-Levy 9 in 1994; it is also illustrated by the discovery of crater chains on Callisto 
and Ganymede (Schenk et al. 1996 examined 116 craters in 11 crater chains). Of course, not every planetary system will have the same comet flux as ours, due to different formative histories and giant planet configurations. The second point leads to a more rapid loss of an atmosphere for a satellite without a strong magnetic field and is a threat to surface life. The third point leads to greater day/night temperature swings. For environments similar to the Galilean satellite system, tidally locked orbits can be beneficial in that they generate internal heat through tidal stressing, but orbital changes on timescales of $10^{8}$ to $10^{9}$ years prevents tides from being a continuously available source of heat (Greenberg et

al. 2000). The problems noted above are somewhat mitigated if the satellite orbits far from its host planet, but in such a configuration the insolation from the parent star becomes more variable. Finally, it is not clear if a giant planet can end up at $\sim 0.5-5$ AU from its host ( $\mathrm{G}-\mathrm{K}$ spectral type) star in an orbit as nearly circular as the Earth's (the present eccentricity of the Earth's orbit is 0.017). To date, all the giant planets found around other solar type stars beyond $\sim 0.15$ A.U. have much more eccentric orbits than does the Earth, except HD 27442, which has an eccentricity of 0.025 (Butler et al. 2001); even Jupiter has an eccentricity of 0.048. Therefore, since they are inferior habitats for complex life, we will not consider natural satellites further.

\subsection{Planet Mass}

It is likely that planet mass is the single most important factor in building a habitable terrestrial planet. We make the simplifying assumption that a terrestrial planet's mass is determined primarily by the local surface density, $\sigma$, of the protoplanetary disk from which it forms. Following the equations describing planet formation in Lissauer (1995), we assume that terrestrial planet mass scales with $\sigma^{1.5}$. This dependence is determined both by the surface density of solids in a feeding zone and the narrower width of a feeding zone 
of a smaller planet. The complexities of planet formation may cause significant deviations from this simple functional dependence, but we believe that this relationship will provide a reasonably close estimate of typical planetary mass. We also assume that: 1) the surface density at a radial location in a protoplanetary disk is directly proportional to the heavy element abundance of the interstellar cloud out of which a planetary system condenses, and 2) the total mass of terrestrial planets that form in a given planetary system is scalable from the Solar System in proportion to the relative heavy element abundances of their parent stars. Until the results of simulations which implicitly include the composition of the birth cloud are made available, we will have to make do with these assumptions.

Present observations of extra-solar-system planets can be of some help to us in determining some astrophysical constraints on habitability. The most important findings to date are: 1) the high mean metallicityf of stars with planets compared to the general field stars, 2) the very short orbital periods of some planets, and 3) the high eccentricities of planets with orbital periods greater than about two weeks (Butler et al. 2000). Combining the first point above with the lack of detection of giant planets in the globular cluster 47 Tucanae (Gilliland et al. 2000) implies that a minimum metallicity near $40 \%$ solar is required to build giant planets. Among the stars with close-in planets, the metallicities are particularly high. This could be due to disk material falling onto the star and polluting its atmosphere, and/or a metallicity dependence on giant planet formation, and/or to a metallicity dependence of planet migration (see Gonzalez et al. 2001 for additional discussion on these points). If the latter is the case, then planet migration may occur

\footnotetext{
${ }^{1}$ Although the preferred term in the literature is "extrasolar", we will employ the more grammatically correct "extra-solar-system" in the present paper.

${ }^{2}$ Note, throughout this paper, we employ the astronomical definition of metals, i.e., element heavier than He.
} 
more frequently in metal-rich systems, leading to the disruption of the orbits of any terrestrial planets in the habitable zone. Similarly, if the high eccentricities of the orbits of the giant planets observed around other stars are due to planet-planet interactions (see Weidenschilling and Marzari 1996), this phenomenon, too, is more likely to occur in metal-rich systems. The metallicity dependencies of these phenomena are not yet well-constrained, so we will not include them in the present study (for an attempt to treat these phenomena quantitatively, see Lineweaver 2000).

It is useful to consider the sensitivity of habitability to changes in planet mass. Lewis (1998) notes three important differences between the Earth and a smaller or larger sibling: 1) heat flow and associated geophysical processes, 2) volatile inventory, and 3) atmosphere loss rate. He estimates that an Earth-like planet identical in composition to the Earth with the same orbit but with one-tenth the mass (about half the radius) would have a lithosphere over twice as thick, suffer much more rapid loss of atmosphere from impact explosive blowoff and dissociative recombination in the exosphere, and have an ocean only $20 \%$ as deep. At the opposite extreme, a planet with 12 times the mass (about twice the radius) would have a much thinner lithosphere, suffer no significant loss of its atmosphere, and have an average ocean depth over three times as great as the Earth's (with no dry land). These estimates assume that the volatile reservoir is primarily the result of outgassing and that its efficiency is proportional to the size of the planet.

There are a couple of other size-dependent factors, which Lewis does not discuss. One concerns impact probability. It is proportional to the square of a planet's the radius (plus a little more owing to gravitational focusing). Furthermore, the increased impact rate would apply to all size scales of impactors; very rare, very large impacts would occur more often on a larger planet. However, the impact energy on a larger planet is diluted over an area proportional to the square of its radius. A proper evaluation of the net dependence of 
impacts on planetary size will require careful consideration of the details of the relevant

processes (such as the mass function of the impactor population). Another size-dependent factor is the time to buildup $\mathrm{O}_{2}$ in the atmosphere (McKay 1996). It should take more time to oxygenate the atmosphere of a larger terrestrial planet, due to its larger inventory of reductants. However, a complete analysis of this factor has yet to be published. For the purposes of the present discussion, we follow Lewis (1998) and require that a terrestrial planet with the same orbit and formation history of the Earth have a mass between one third and three Earth masses to be habitable, though it is our opinion that this range is probably too broad.

We note that the above discussion must be placed in the broader context of the terrestrial planet environment. In particular, a cloud that is sufficiently metal-rich to form giant Earth siblings will also likely form smaller terrestrial planets (cf. Wetherill 1996). Therefore, the problems noted above for a very large terrestrial planet in a given system would still not prevent that system from having a terrestrial planet in the required mass range. The same cannot be said of a metal-poor cloud, which forms only small terrestrial planets. Given this, then, habitability for a given system cannot be excluded solely on the basis that it forms one or more massive Earths, but it might be excluded on other grounds related to high initial metallicity (as noted earlier).

\subsection{Plate Tectonics}

Of the geophysical processes that affect habitability, plate tectonics is probably the most critical, because it plays a central role in maintaining earth's long-term climate stability (Kasting et al. 1993; Franck et al. 1999, 2000a). Subduction of carbonates, and their ultimate thermal decomposition, provides the volcanic $\mathrm{CO}_{2}$ source that drives the $\mathrm{CO}_{2}$-rock cycle. The continuing $\mathrm{CO}_{2}$ supply from subducted sediments combined with the 
temperature dependent removal of $\mathrm{CO}_{2}$ by chemical weathering provides negative feedback that opposes major global temperature variations. Without plate tectonics it is unlikely the earth or similar planets could maintain habitable surface environments for animal life for long periods of time (Franck et al. 1999, 2000a).

Unfortunately, the requirements for the origin and maintenance of plate tectonics are not well known. On earth the process produces spreading center ridges, trenches, subduction zones and linear mountain ranges. From the lack of similar features on other bodies it is clear this process is not currently operating on any other solar system body. Mars and Venus display a rigid lid, and lack plate tectonics at the present (Tackley 2000). Mars is apparently too small. Venus, a near twin of Earth, does not have plate tectonics probably because of its lack of water, which lowers the melting points of magmas and promotes general ductility required for subduction (Karato and Jung 1998). A necessary, though certainly not sufficient, requirement for plate tectonics is a long-term supply of heat. Radioactive decays of ${ }^{235,238} \mathrm{U},{ }^{232} \mathrm{Th}$, and ${ }^{40} \mathrm{~K}$ are considered to be the primary sources of heat in the Earth's interior. Very early in the Earth's history there were other sources of interior heat: short-lived radioisotopes, heat of formation (from accretion, including the lunar-forming impact), and formation of the core. However, after the first 1.5 Gyrs, the initial thermal state of the Earth was erased due to a re-adjustment effect in mantle convection (see Franck 1998). Therefore, following an early settling-down period, the heat flow through a terrestrial planet's mantle is largely determined by the abundances of long-lived radioactive isotopes in its interior. However, the amount of volatile outgassing does depend rather sensitively on the initial thermal state (Franck 1998); hence, the amount of outgassing will be dependent on the details of the formation process. 


\subsection{CHZ Limits}

The dimensions of the Circumstellar Habitable Zone (CHZ) in the Solar System are defined by more than just the flux of stellar radiation. There are several other parameters that should be included in the definition if it is to be generalized to any location and time in the Milky Way. The relevant parameters are: mass, composition, orbit, and type of parent star. Taken together, the possible parameter space covered by these quantities is very large, and it is not possible to set separate constraints on them. For example, the inner edge of the CHZ for a terrestrial planet smaller than the Earth would be farther from the Sun than the $\mathrm{CHZ}$ of the Earth due to the more rapid loss of its atmosphere. However, there are a few simplifying assumptions we can make to narrow it significantly. One is the restriction of the orbit to small eccentricity in order to prevent large temperature swings. Another one is the restriction to main sequence stars similar in spectral type to the Sun (early G), which finds support from the Weak Anthropic Principle (Gonzalez 1999b) and theoretical arguments concerning stellar lifetimes and tidal locking and observations of flare activity in low mass dwarfs.

There are at least three other phenomena/processes that are presently not included in studies of the CHZ, such as those cited in the Introduction. One is the variation of the impact threat with position, due to the increase in encounter velocities with decreasing distance from the parent star. The impact energy of an asteroid or comet is proportional to the square of the relative velocity between the impactor and the planet. Another factor is the composition gradient of the early solar nebula. Lewis (1997) describes quantitatively the condensation sequence of elements as a function of distance from the central star (described in greater detail below). A third factor concerns the details of planet formation. For example, the radial distribution of terrestrial planets is stochastic and is relatively insensitive to stellar mass according to simulations of the late stages of planet formation 
carried out by Wetherill (1996). Taken together, these additional factors more severely constrain the dimensions of the CHZ. However, a consideration of the complex relationships among the various factors defining the $\mathrm{CHZ}$ are beyond the scope of the present work. We, instead, focus on a subset of these, which we will explore within the larger context of the Milky Way.

\subsection{Composition}

In addition to the overall metallicity (and hence mass) there are compositional factors that influence habitability. A comparison between Earth and Venus illustrates that planet mass is not the only factor that determines a planet's geophysics. Although they are nearly the same mass, Earth and Venus have very different geology. Most of these differences probably date back to the different origins of Earth and Venus. Lewis (1997) notes that the composition gradient of the early solar nebula inferred from the expected radial condensation sequence of the elements will result in planetary embryos of varying composition. The early stages of planet formation are likely to draw from a relatively narrow range in distance from the central star, but latter accretion is likely to be from a relatively larger range (Wetherill 1996). This implies that the properties of a terrestrial planet's core and mantle depend rather sensitively on its place of formation. For example, more FeS should have condensed in the proto-Earth relative to the proto-Venus, and this would have lead to a significant reduction in the melting point of the Earth's core. The relative abundance of ${ }^{40} \mathrm{~K}$, an important source of long-term heat, would also have been variable; given its relatively high volatility, less of it would have been incorporated by protoplanets inside the Earth's orbit. The CHZ definition, then, must include not only the dependence of solar insolation but also of bulk composition on radial distance. The detailed dependencies of planetary composition on place of formation are beyond the scope of this 
paper, but it is a factor that must be kept in mind when we discuss Galactic chemical evolution.

The most abundant element in the Earth by number is O, while the most abundant element by mass is Fe (Kargel and Lewis 1993). The next most abundant terrestrial elements are $\mathrm{Mg}$ and $\mathrm{Si}$; the cosmically abundant elements, $\mathrm{H}, \mathrm{He}, \mathrm{C}, \mathrm{N}$, are present only in trace amounts (Kargel and Lewis 1993). The O abundance can be considered as a free parameter, since it forms oxides with $\mathrm{Mg}, \mathrm{Si}$, and Fe. Another factor likely to be relevant to the habitability of a terrestrial planet is the abundance of $\mathrm{Mg}$ and Si relative to Fe, which will determine the mass of the core relative to the mantle. The condensation temperatures of $\mathrm{Mg}$, Si, and $\mathrm{Fe}$ are similarf, so they should condense in ratios similar to that of their host star and terrestrial planets should experience relatively little differential fractionation. In the Milky Way's disk, stars with low $\mathrm{Fe} / \mathrm{H}$ have relatively larger $\mathrm{Mg} / \mathrm{Fe}$ and $\mathrm{Si} / \mathrm{Fe}$ ratios (see Section 3.1.1). These ratios can differ by up to 250 percent from the solar values and may have major influence on the interior workings of a terrestrial planet, such as the efficiency of core formation, the nature of solid-state convention, and its mineralogy.

Gaidos (2000) has noted the likely significance of the $\mathrm{C} / \mathrm{O}$ ratio in the formation of planets. The $\mathrm{C} / \mathrm{O}$ ratio determines how much oxygen in an accretion disk is in $\mathrm{CO}$ and how much is in water. Water ice was an abundant condensable substance in the outer regions of our Solar System's early history and was an important ingredient in the formation of the embryos of the giant planets. As discussed above, it is also an essential ingredient in forming a habitable terrestrial planet. The bulk $\mathrm{C} / \mathrm{O}$ ratio in a planet will be the result of a variety of fractionation and volatile delivery processes, so one cannot just equate it to the initial $\mathrm{C} / \mathrm{O}$ ratio in a protoplanetary nebula. The relevance of the $\mathrm{C} / \mathrm{O}$

\footnotetext{
${ }^{3}$ Lodders and Fegley (1998) list the condensation temperature of Mg, Si, and Fe as 1340, 1340, and $1337 \mathrm{~K}$, respectively.
} 
ratio for the formation of habitable terrestrial planets is probably less direct, affecting on the characteristics of the giant planets and the population of cometary bodies. However, Gonzalez et al. (2001) do not detect a significant difference in the $\mathrm{C} / \mathrm{O}$ ratio between the parent stars of extra-solar-system planets and the general field star population (the previously reported low $\mathrm{C} / \mathrm{O}$ ratio for the Sun compared to field stars has proven to be

spurious, due to an unrecognized systematic error in $\mathrm{C}$ abundance estimates from one of the cited studies). Therefore, support for the importance of the $\mathrm{C} / \mathrm{O}$ ratio to planet formation currently comes only from theoretical arguments.

\subsection{Basic Requirements}

In summary, sufficient metals must be available in the interstellar medium to build a habitable terrestrial planet. Following Lewis (1998), we assume its mass should be between approximately one third and three Earth masses. In addition, the relative proportions of $\mathrm{Si}, \mathrm{Mg}$, and Fe should be similar to those in the Earth, and there are minimum required abundances of the long-lived radioactive isotopes, ${ }^{235,238} \mathrm{U},{ }^{232} \mathrm{Th}$, and ${ }^{40} \mathrm{~K}$.

The abundance of ${ }^{40} \mathrm{~K}$ in a terrestrial planet's crust may be relevant also to the origin of life. Draganic et al. (1991) noted that the ionizing radiation produced from the decay of ${ }^{40} \mathrm{~K}$ in the oceans of the early Earth would have generated $\mathrm{O}_{2}$ and $\mathrm{H}_{2} \mathrm{O}_{2}$ via radiolysis of water. The early stages of the extant chemical evolution of life scenarios require reducing conditions, so a planet with too much ${ }^{40} \mathrm{~K}$ mixed in its oceans may delay or prevent the appearance of life.

\section{GALACTIC EVOLUTION AND SPATIAL DISTRIBUTION OF THE ELEMENTS}




\subsection{The Stable Elements}

Since the formation of the Milky Way about 12 to 15 Gyr ago, the metals have increased in abundance in the interstellar medium (ISM) near the Sun from near zero to about $2 \%$ by mass. To first order, the cosmic abundances of the elements heavier than Boron scale with Fe, which has many easily measured absorption lines in the spectra of Sun-like stars. Therefore, what is sometimes termed the "metallicity" of a star is often its Fe abundance - in the following we will employ the terms $[\mathrm{Fe} / \mathrm{H}]]^{\mathrm{H}}$ and metallicity interchangeably. Observational and theoretical knowledge of the evolution of the chemical element abundances in the Milky Way has improved greatly over the last 30 years. The overall abundance trends of most elements are now well established from spectroscopic observations of stars in the solar neighborhood (e.g., Gratton et al. 2000; Chen et al. 2000; reviewed by McWilliam 1997). In the following we will consider the evolution of both the mean $\mathrm{Fe}$ abundance and abundance ratios, as $\mathrm{X} / \mathrm{Fe}$, and discuss their relation to habitability as defined in Section 2.

The chemical evolution of the ISM is governed primarily by the rate of infall of unprocessed gas and processing of ISM matter by massive and intermediate mass stars (Timmes et al. 1995; Pagel 1997; Portinari et al. 1998; Samland 1998). The primary

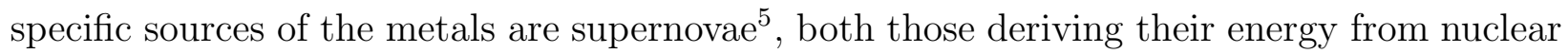
explosions of white dwarfs (type Ia) and those deriving their energy from core collapse (type

\footnotetext{
${ }^{4}[\mathrm{X} / \mathrm{Fe}] \equiv \log \left(N_{\mathrm{X}} / N_{\mathrm{Y}}\right)_{\mathrm{Star}}-\log \left(N_{\mathrm{X}} / N_{\mathrm{Y}}\right)_{\text {Sun }}$, where the variable $\mathrm{N}$ corresponds to the number density abundance of element $\mathrm{X}$ and $\mathrm{Y}$. Hence, a star with $[\mathrm{Fe} / \mathrm{H}]=+0.5$ has 3 times the $\mathrm{Fe} / \mathrm{H}$ abundance ratio of the Sun.

${ }^{5}$ Except $\mathrm{C}$ and $\mathrm{N}$, for which a substantial contribution is from intermediate mass stars.
} 
Ib,c; type II). [] SNe II are the primary source of O, the $\alpha$ ], and the $r$-process elements, and SNe Ia are the primary source of the Fe-peak (i.e., Fe, Co, Ni) elements (Timmes et al. 1995; Portinari et al. 1998; Samland 1998). In the simplest terms, the evolution of abundance ratios can be understood in terms of the changing relative contributions from these two basic SN types, which can be due to changes in the initial mass function and in the star formation rate. A particularly important constraint on Galactic chemical evolution models is the observed decline in the $\mathrm{O} / \mathrm{Fe}$ ratio with increasing $\mathrm{Fe} / \mathrm{H}$ among nearby stars (e.g., Edvardsson et al. 1993). This is thought to result from the rise in the frequency of SNe Ia with time relative to that of SNe II (Mathews et al. 1992; see Fig. 39 of Timmes et al. 1995, Fig. 12 of Portinari et al. 1998, and Fig. 1 of Samland 1998).

When discussing chemical evolution, it is convenient to divide up the Milky Way into four components: thin disk, thick disk, bulge, and halo. While they partially overlap in space, these components can be relatively well separated with the addition of data on stellar kinematics. The thin disk, of which the Sun is a member, includes significant ongoing star formation activity, and its kinematics is more nearly purely rotational than the other components; stars extend about 180 pc on either side of the midplane. The thick disk is basically a fatter version of the thin disk and contains stars that are more metal-poor than it and extend about $600 \mathrm{pc}$ on either side of the midplane. The bulge is centered at the center-of-mass of the Milky Way.] Its constituent stars display a large range in metallicity. In the Milky Way the bulge experienced an early rapid rate of star formation, which has since slowed, but continues to the present day. The halo is a spherical distribution of

\footnotetext{
${ }^{6}$ Hereafter, SNe Ib,c II will be grouped together as SNe II.

${ }^{7}$ The $\alpha$ elements typically measured in stellar spectra are $\mathrm{Mg}, \mathrm{Si}, \mathrm{Ca}$, and $\mathrm{Ti}$.

${ }^{8}$ Here we are assuming that the thin and thick disks each has a central hole. Therefore, given this assumption, only bulge stars occupy the central region of the Milky Way.
} 
individual stars and globular clusters extending to about $100 \mathrm{kpc}$. It is the oldest, most metal-poor component. We discuss each of these components separately below.

\subsubsection{The Thin Disk}

The overall metallicity of the ISM in the thin disk has been increasing steadily (but episodically on short timescales; Rocha-Pinto et al. 2000). Gonzalez (1999a) derived the following equation from a sample of nearby $\mathrm{F}$ and $\mathrm{G}$ dwarfs with well-determined physical parameters, relating $[\mathrm{Fe} / \mathrm{H}]$ to age and to mean Galactocentric distance, $\mathrm{R}_{m}$ :

$$
[F e / H]=(-0.01 \pm 0.02)-(0.07 \pm 0.01)\left(R_{\mathrm{m}}-R_{0}\right)-(0.035 \pm 0.005) \tau
$$

where $\tau$ is the age in Gyr and $\mathrm{R}_{0}$ is the present Galactocentric distance of the Sun (we adopt $R_{0}=8.5 \mathrm{kpc}$ in the present study). Since this equation is determined from a sample of nearby stars, it is not applicable to the halo or to the bulge of the Milky Way, which have had significantly different histories from the local disk. The assumption of a linear dependence of $[\mathrm{Fe} / \mathrm{H}]$ on age is justifiable for stars no older than about $80 \%$ of the age of the Milky Way; beyond that, $[\mathrm{Fe} / \mathrm{H}]$ drops off very rapidly with age (see Fig. 13 of Portinari et al. 1998). The zero point of Eq. 1 is consistent with observations of nearby young solar type stars, B stars, and H II regions. The present radial abundance gradient in the thin disk, $-0.07 \mathrm{dex} \mathrm{kpc}^{-1}$, is well determined from observations of several different kinds of objects in the Milky Way (e.g., B stars, H II regions, open clusters, and young solar-type stars - see Rolleston et al. 2000 and references cited therein); the uncertainty in the slope given in Eq. 1 is based on B star data. Its magnitude does vary among different galaxy types (see Henry and Worthey 1999). Rolleston et al. (2000) also note that their data are consistent with a single slope, as opposed to a sharp break near $10 \mathrm{kpc}$ from the Galactic center as some 
have argued previously. Hou et al. (2000) present theoretical estimates of the evolution of radial abundance gradients in the Milky Way. Unfortunately, since observations can only give us the present values of the Galactic abundance gradients, estimates of their evolution are rather model dependent. Given this uncertainty and the fact that it is a second-order effect, we will ignore possible evolution of the gradients in the present study.

In addition to the abundance gradient in the disk, we must also consider the spread in $[\mathrm{Fe} / \mathrm{H}]$ at a given age and $\mathrm{R}_{m}$. The magnitude of the spread affects the degree to which the metallicity gradient is an important factor for habitability. If the spread is large in relation to the magnitude of the gradient, then the gradient is largely washed out and loses its relevance for the radial dependence of habitability in the Milky Way's disk. A large intrinsic spread in the $[\mathrm{Fe} / \mathrm{H}]$ value of newborn stars at a given $\mathrm{R}_{m}$ will result in the formation of some stars with a given value of $[\mathrm{Fe} / \mathrm{H}]$ earlier than indicated by Eq. 1. If, however, the spread is small, then the radial metallicity gradient has a more important role in habitability. The value of the spread in $[\mathrm{Fe} / \mathrm{H}]$ is still a matter of some controversy. One of the most often cited studies is that of Edvardsson et al. (1993), who performed a spectroscopic survey of nearly 200 nearby solar-type stars. They found a dispersion in $[\mathrm{Fe} / \mathrm{H}]$ at a given age of about 0.25 dex, which is much larger than observational error alone can explain. Wielen et al. (1996) modeled the observed scatter in $[\mathrm{Fe} / \mathrm{H}]$ (using the dataset of Edvardsson et al. 1993) within the framework of a stellar orbital diffusion model. Using an improved dataset based largely on the Edvardsson et al. (1993) sample, Gonzalez (1999a) confirmed the large metallicity spread.

Rocha-Pinto et al. (2000) give an independent estimate of the metallicity dispersion in the solar neighborhood with a different sample, finding a value about half of the above-cited value. It is significant that Rocha-Pinto et al.'s (2000) estimate of the spread is less than that derived by others. This implies that their sample is more reliable, since the 
total measured spread is the sum of measurement errors, unmodelled biases, and intrinsic cosmic scatter; however, it is still possible that an unrecognized systematic bias in their analysis may have yielded an artificially small scatter. Rocha-Pinto et al. (2000) attribute the difference largely to selection biases that went into the preparation of the original Edvardsson et al. (1993) sample. Based on spectroscopic analyses of young F and G dwarfs, Gonzalez (1999a) and Gaidos and Gonzalez (2000) find that the initial dispersion in [Fe/H] is about 0.08 dex; the cosmic dispersion must be less than this value, as the measurement error is similar in magnitude. Certainly the last word has not been said on this topic; additional observations are required to firmly establish the cosmic dispersion.

Since our interest in this section is to estimate the probability of forming Earth mass terrestrial planets in the disk of the Milky Way at any time and place, the relevant dispersion value in $[\mathrm{Fe} / \mathrm{H}]$ is that at the time of formation of a given star. For the purpose of the present discussion, then, we will adopt a time-constant dispersion in $[\mathrm{Fe} / \mathrm{H}]$ of 0.08 dex. We show in Figs. 1 and 2 graphical representations of Eq. 1, assuming that terrestrial planet mass scales as $10^{1.5[\mathrm{Fe} / \mathrm{H}]}$ (see Section 2.1). The equation has not been extrapolated to times within 3 Gyr of the formation of Milky Wayg or within 2 kpc of the Galactic center due to the breakdown of Eq. 1 in these regimes. As can be seen in Fig. 2 solar metallicity was reached in the ISM in early times at smaller $\mathrm{R}_{m}$.

Figs. 1 and 2 should only be considered as rough approximations for reasons already given. Another one is the assumption of constant dispersion in metallicity with time. The cosmic dispersion in metallicity (and in abundance ratios) was much greater in the early history of the Milky Way. This is a consequence of the effect on ISM abundances of individual SNe. During the early rapid buildup of metals, the metal-poor ISM was more

\footnotetext{
${ }^{9}$ Note, in the present work we assume that the first metals were incorporated into the Milky Way were formed 15 Gyr ago.
} 
sensitive to the stochastic nature of inhomogeneously distributed SNe. As the metallicity of the ISM increased, individual SNe lead to smaller fractional increases of the metallicity (and smaller changes to the abundance ratios). This effect is seen in the abundance patterns of the most metal-poor stars in the halo. However, the large observed scatter in abundances among the oldest stars in the Milky Way halo is not relevant to the present discussion, as their metallicities are too low to form terrestrial planets. At later times the abundance variations can be large for another reason. As the Milky Way ages, the gas mass decreases, and hence, becomes more sensitive to individual SNe again.

We argued in Section 2 that certain elements must be present in the right proportions to build an Earth-substitute with similar geophysics. Specifically, the C/O, Mg/Fe, Si/Fe, and $\mathrm{S} / \mathrm{Fe}$ ratios are likely to be relevant to the habitability of a terrestrial planet. Of these, high quality observational data exist for all but S/Fe. Below we discuss the observed trends in these ratios among thin disk stars.

There is relatively little published observational material on the $\mathrm{C} / \mathrm{O}$ ratio. Garnett et al. (1999) presented C and O abundances for the nearby spiral galaxies, M101 and NGC 2403. They find radial gradients near $-0.05 \mathrm{dex} / \mathrm{kpc}$, though their results are of a preliminary nature, given the small number of data points in their sample. Observations of nearby solar-type stars show a significant increase in the $\mathrm{C} / \mathrm{O}$ ratio with $\mathrm{Fe} / \mathrm{H}(\sim 0.23$ dex/dex) and, hence, with time. The chemical evolution models of Carigi (1996) imply that the slope of the $\mathrm{C} / \mathrm{O}$ ratio with metallicity has steepened greatly in the last few Gyrs, finding a value consistent with the observations. Carigi (1996) also finds that the C/O gradient in disk galaxies is negative, consistent with the observations of Garnett et al. (1999).

Given these trends, the formation of a planetary system with a $\mathrm{C} / \mathrm{O}$ ratio equivalent to that of the Sun will occur at a specific time for a given $\mathrm{R}_{m}$. If a low $\mathrm{C} / \mathrm{O}$ ratio is required to 
build habitable planetary systems, as was argued in Section 2, then stars born in the solar neighborhood in the future might not be accompanied by them. Without knowing what range of $\mathrm{C} / \mathrm{O}$ is required for habitability, we cannot quantify its effect on the habitable zone in the Milky Way, but observations of the parent stars of extra-solar-system planets might eventually allow us to better constrain it.

The $\mathrm{Mg} / \mathrm{Fe}$ and $\mathrm{Si} / \mathrm{Fe}$ ratios are observed to be declining with increasing $\mathrm{Fe} / \mathrm{H}$ among disk stars with slopes near -0.3 and $-0.1 \mathrm{dex} / \mathrm{dex}$, respectively. Therefore, in the future terrestrial planets will form with relatively larger iron cores.

\subsubsection{The Thick Disk, Bulge, and Halo}

The thick disk has properties intermediate between the halo and the thin disk. Its constituent stars are only slightly younger that the halo but substantially older than the thin disk. The thick disk population has a distinct chemical history compared to the thin disk. The stars have a mean $[\mathrm{Fe} / \mathrm{H}]$ value near -0.6 dex, and most $\alpha$-elements (specifically $\mathrm{O}, \mathrm{Mg}, \mathrm{Si}, \mathrm{Ca}, \mathrm{Ti}$ ) have $[\mathrm{X} / \mathrm{Fe}]$ values 0.2 to 0.3 dex above solar (Gratton et al. 2000; Prochaska et al. 2000). The most extreme members reach nearly solar $[\mathrm{Fe} / \mathrm{H}]$, but most $\alpha$-element abundances are relatively constant throughout the observed range in $[\mathrm{Fe} / \mathrm{H}]$. Hence, if our assumptions from Section 2 are correct, then a terrestrial planet that forms around a thick disk star is smaller and has a smaller iron core relative to its mantle compared to the Earth; the typical terrestrial planet around a thick disk star should have about $13 \%$ the mass of the Earth. However, a small fraction of thick disk stars might be accompanied by an Earth-mass terrestrial planet.

The bulge of the Milky Way (or of any other spiral galaxy) is not merely an extrapolation of the disk or halo; it is a distinct component. Star formation activity in the 
bulge peaked earlier than in the disk, but it has continued up through the present (Moll et al. 2000). Owing to the different chemical evolution history of the bulge compared to the disk, the abundance ratios among its stars are different from those among stars in the solar neighborhood. Spectroscopic observations by Rich and McWilliam (2000) show that $[\mathrm{Fe} / \mathrm{H}]$ ranges from -1.6 to +0.55 dex, with the peak near -0.2 dex. In addition, they find $[\mathrm{X} / \mathrm{Fe}]$ values near +0.2 to 0.3 dex near solar $[\mathrm{Fe} / \mathrm{H}]$ for the $\alpha$-elements, $\mathrm{O}, \mathrm{Mg}$, Si, and Ti. Globular clusters found in the bulge appear to share the composition characteristics of the bulge stars. Therefore, Earth-mass terrestrial planets should be common in the bulge, but, as with thick disk stars, they likely have relatively small iron cores.

Halo stars span a wide range in metallicity, from $[\mathrm{Fe} / \mathrm{H}]=-4$ to just below solar, with the peak near -1.5 ; there is also a trend of decreasing scatter in the abundances ratios (expressed as X/Fe) with increasing metallicity (see review by Norris 1999). Star formation was active only during the first couple of Gyrs in the halo. The kinematics result in mostly eccentric, plunging orbits, which bring stars close to the Galactic center and pass through the disk at high velocity (over $200 \mathrm{~km} / \mathrm{sec}$ ). The halo also contains about 150 globular clusters, which share the same metallicity distribution as the halo field stars, except for the absence of globular clusters with $[\mathrm{Fe} / \mathrm{H}]<-2.5$. Therefore, on its chemical characteristics alone, the halo is very unlikely to contain habitable terrestrial planets.

\subsubsection{Summary of $\mathrm{C}, \mathrm{O}, \mathrm{Mg}, \mathrm{Si}, \mathrm{Fe}$}

In summary, each of the four components of the Milky Way is characterized by a distinct composition, resulting from a distinct star formation history. The precise composition of the Sun is the result of a history of chemical enrichment of the thin disk of the ISM not likely to be found in the other components, though cosmic scatter at a given metallicity will lead to some overlap. Even within the thin disk, radial abundance gradients 
(somewhat analogous to the condensation sequence gradient in the early solar nebula) ensure that stars with the solar composition are unlikely to form far from the solar circle. Just how large a difference in composition can be tolerated in forming an Earth-substitute we cannot yet answer, though.

\subsection{The Long-Lived Radionuclides}

\subsubsection{Evolution of ISM Abundances}

Second in importance for building terrestrial planets, after the mean metallicity of the ISM, is likely to be the relative abundances of the long-lived radioisotopes. The heat released from the decay of long-lived radioactive isotopes is believed to be the primary source of internal heat in the present Earth. The most important heat-producing isotopes are ${ }^{40} \mathrm{~K},{ }^{232} \mathrm{Th},{ }^{238} \mathrm{U}$, and ${ }^{235} \mathrm{U}$ (Fowler 1990; Kargel and Lewis 1993). The present abundances of these isotopes have been well determined in primitive meteorites (Anders and Grevesse 1989). Outside the Solar System, only the atomic abundances of K and Th can be determined from photospheric stellar spectra. Since ${ }^{232} \mathrm{Th}$ is the only long-lived isotope of this element, its abundance can be equated with the spectroscopically-derived

atomic value; this cannot be done with $\mathrm{K}$. We will address the more difficult case of ${ }^{40} \mathrm{~K}$ later in this section.

The isotopes of Th and $\mathrm{U}$ are produced only by the $r$-process, and SNe II are believed to be their main production sites (Mathews et al. 1992; Timmes et al. 1995; Cowan et al. 1999). The half-lives of ${ }^{232} \mathrm{Th},{ }^{235} \mathrm{U}$, and ${ }^{238} \mathrm{U}$ are $14.05,0.7038$, and $4.468 \mathrm{Gyr}$, respectively. We begin by calculating the evolution of the $\mathrm{Th}$ and $\mathrm{U}$ abundances relative to Fe in the local ISM. We employ Fe as the reference element for two reasons: 1) Fe is the most abundant element in the Earth by weight, and 2) Fe, Th, and U have similar condensation 
temperatures (Lewis 1997). Therefore, Fe, Th, and U are likely to experience fractionation to similar degrees during the early stages of planet formation in a protoplanetary disk.

For the purpose of employing theoretical Galactic chemical evolution models, it is useful first to compare the evolution of the $\mathrm{Th}$ and $\mathrm{U}$ abundances to that of a stable $r$-process element. This procedure is advantageous since these species are produced by the same source, and there is some empirical evidence that the $r$-process abundance pattern is universal (Cowan et al. 1999). Assuming it is, the ratio of the abundance of a radioactive $r$-process element to that of a stable $r$-process element will have only a weak dependence on the details of Galactic chemical evolution. The best such reference element is Eu, because it is easy to measure in stellar spectra, and nearly all of it comes from the $r$-process (all but $3 \%$ in the Solar System according to Cowan 1999). We can determine the Eu/Fe ratio from observations and convert it to $\mathrm{Th} / \mathrm{Fe}$ and $\mathrm{U} / \mathrm{Fe}$ ratios by applying theoretical $\mathrm{Th} / \mathrm{Eu}$ and $\mathrm{U} / \mathrm{Eu}$ ratios, respectively.ण0

We display in Fig. 3 (panel a) the [Eu/Fe] estimates for a sample of 72 nearby single $\mathrm{F}$ and G dwarfs from the spectroscopic study of Woolf et al. (1995); this is currently the best such sample. We have fit a simple linear equation to the data:

$$
[E u / F e]_{c}=(-0.005 \pm 0.013)-(0.282 \pm 0.039)[F e / H]
$$

where $[\mathrm{Eu} / \mathrm{Fe}]_{c}$ is the value of $[\mathrm{Eu} / \mathrm{Fe}]$ corrected to the solar $\mathrm{R}_{m}$ by adding the term, 0.11( $R_{m}$ - 8.8), which was determined from a multiple linear regression fit to the data with $\mathrm{R}_{m}$ and $[\mathrm{Fe} / \mathrm{H}]$ as independent parameters. A second-order fit would describe the data slightly better, but for the present application, a first-order fit is sufficient (see Fig.

10 There are measurements of the Th/Fe ratio in a few nearby solar type stars, but they are not considered very reliable, and so we will not make use of them. 
3, panel b). When combined with Eq. 1 with $\mathrm{R}=\mathrm{R}_{m}$, Eq. 2 gives us a $[\mathrm{Eu} / \mathrm{Fe}]_{c}$ - age relation (note that the timescale for this relation is based on stellar evolution theory, not nucleocosmochronology).

Next, we calculate $\left[{ }^{232} \mathrm{Th},{ }^{235} \mathrm{U},{ }^{238} \mathrm{U} / \mathrm{Eu}\right]$ - age relations. In this case, observations are very sparse, but theory can help us. We make use of Clayton's (1988) Eqs. (13) and (14), which give the abundances of stable and radioactive species in the ISM as a function of time. The equations are based on a Galactic infall model with three adjustable parameters: $k, \omega$, and $\Delta$. We reproduce the equations below:

$$
Z-Z_{0}=\frac{y \omega \Delta}{k+1}\left[\frac{t+\Delta}{\Delta}-\left(\frac{t+\Delta}{\Delta}\right)^{-k}\right]
$$

and

$$
Z_{\lambda}-Z_{0} e^{-\lambda t}=y \omega e^{-\lambda t}\left(\frac{t+\Delta}{\Delta}\right)^{-k} I_{k}(t, \lambda)
$$

where Eq. 3 applies to stable isotopes and Eq. 4 to radioactive ones. The $\mathrm{I}_{k}(\mathrm{t}, \lambda)$ term is given by Clayton as a function of $t, \Delta$, and $\lambda$ (the radioactive decay rate) for a given $k$. The production yield of a given isotope is denoted by $y$, and the gas consumption rate is denoted by $\omega$. The parameters $\Delta$ and $k$ determine infall rate as well as the disk mass growth rate. We will be working with Eqs. 3 and 4 in ratio form only, so knowledge of $\omega$ is not required for our application.

Pagel and Tautvaisiene (1995) have applied, in slightly modified form, Clayton's model to the observed abundance trends among local disk stars. They derived the following values for the infall model parameters: $k=3, \omega=0.3 \mathrm{Gyr}^{-1}$, and $\Delta=4.33 \mathrm{Gyr}$; in addition, they adopted an age of 15 Gyr for the Milky Way (which is consistent with the timescale of our Eq. 1). These values differ slightly from Clayton's older estimates: $k=1$, 
$\omega=0.3 \mathrm{Gyr}^{-1}$, and $\Delta=1$ Gyr. We will adopt the estimates of Pagel and Tautvaisiene in the following calculations, since they are based on more recent data. We also need the production ratios. Cowan (1999) gives: $\log (T h / E u)_{0}=-0.32, \log \left({ }^{235} U / E u\right)_{0}=-0.48$, and $\log \left({ }^{238} U / E u\right)_{0}=-0.68$ (note, combining the theoretical Th/Eu production ratio with the observed Th/Eu ratio in two very metal-poor stars, Cowan et al. 1999 derive a mean age of $15.6 \pm 4$ Gyr for them).

With this set of parameter values and Eqs. 3 and 4, we have calculated the present abundances of the $\mathrm{Th}$ and $\mathrm{U}$ isotopes relative to $\mathrm{Eu}$ for a star formed at a time, $\mathrm{t}$, after the formation of the Galaxy (Fig. 4). The absolute abundance ratios have been converted into relative ones using the present meteoritic isotopic abundances listed by Anders and Grevesse (1989). The calculations take into account the free decay of the radioactive isotopes in the atmosphere of a star between the time of its formation and the present.⿴囗十 There are a couple of points to notice about Fig. 4: 1) the calculations yield very nearly the Solar System abundances at $\mathrm{t}=10.5 \mathrm{Gyr}$, and 2) the relative abundances are greater in the recently formed stars. The second point is simply due to the fact that the mean age of the radioactive isotopes is less in the younger stars.

The close agreement between theory and observation for two very metal-poor halo stars and for the Sun gives us confidence that the analysis is self-consistent. But, we should note a word of caution concerning comparison of theory with the Solar System abundances. In general, due to the Weak Anthropic Principle, it is dangerous to assume that a particular solar parameter is typical (Gonzalez 1999b). Those studies that use the Solar System Th/r-process ratio to derive an estimate of the age of the Milky Way might therefore be

\footnotetext{
${ }^{11}$ Note that Eqs. 3 and 4 yield the abundance of a given species in the ISM at some time, t, after the formation of the Milky Way. To calculate the free decay of a radioactive isotope between $\mathrm{t}$ and the present, Eq. 4 must be multiplied by $e^{-(15-t) \lambda}$.
} 
incorrect. They should instead use the mean ratio determined from a sample of nearby stars which excludes the Sun. Unfortunately, it is not possible to determine the Th/Eu ratio in the atmosphere of a star as precisely as it can be determined in a meteorite.

To form the $\left[{ }^{232} \mathrm{Th},{ }^{235} \mathrm{U},{ }^{238} \mathrm{U} / \mathrm{Fe}\right]$ - age relations, we can combine the results of Fig. 4 with Eqs. (1) and (2). However, instead of using the results of Fig. 4 directly, it is more appropriate for the present discussion to calculate the abundances of the radioactive elements 4.5 Gyr after the formation of the star. We show the results of such a calculation in Fig. 5. The results indicate that, relative to $\mathrm{Fe}$, all three radioactive isotopes decrease with t. For example, stars born today will, in 4.5 Gyr, have a smaller ratio of Th/Fe than the Sun does today. This is due to the fact that the Fe abundance in the ISM is rising more rapidly than is the Th abundance.

The Galactic chemical evolution of ${ }^{40} \mathrm{~K}$ is more difficult to determine for two reasons: 1) the sources of ${ }^{40} \mathrm{~K}$ are diverse, both $r$ - and $s$-process, and 2) it is not possible to measure the abundance of ${ }^{40} \mathrm{~K}$ in the spectra of stars. The most abundant isotopes of $\mathrm{K}$ in the Solar System are ${ }^{39} \mathrm{~K}$ and ${ }^{41} \mathrm{~K}$, both of which are produced by Type II SNe (Thielemann et al. 1996); stellar spectra only give us the sum of the ${ }^{39} \mathrm{~K},{ }^{40} \mathrm{~K}$, and ${ }^{41} \mathrm{~K}$ abundances. In addition, since $\mathrm{K}$ is more volatile than $\mathrm{U}$ and $\mathrm{Th}$, it will condense out of the protoplanetary nebula at relatively larger radial distances from the parent star. Hence, there is a radial gradient in the radiogenic isotope concentration among planetary embryos; the gradient should be particularly important if significant amounts of $\mathrm{K}$ can be incorporated into the core, which should form early in the accretion process.

The isotopes of the elements, $\mathrm{Se}, \mathrm{Kr}, \mathrm{Rb}$, and $\mathrm{Sr}$, are likely to be produced in the same site as ${ }^{40} \mathrm{~K}$ (Prantzos et al. 1990; Podosek et al. 1999). They belong to the so-called weak $s$-process component, i.e., the one with the smallest neutron exposure, of the Solar System isotopic distribution, which are thought to be produced in the He-burning regions 
of massive stars (Käppeler et al. 1989; Prantzos et al. 1990). Some ${ }^{40} \mathrm{~K}$ is also produced in SNe II (Thielemann et al. 1996), but the primary production is probably by the $s$-process. According to Cowan (1999), the $s$-process contribution of K is $89 \%$ in the Solar System. Thus, although ${ }^{40} \mathrm{~K}$ is produced by more than one mechanism, it is probably dispersed into the ISM by SNe II (note that we are not saying that ${ }^{40} \mathrm{~K}$ is generated in a SN II event, but, rather, that the ${ }^{40} \mathrm{~K}$ produced previously in the interior of the SN progenitor is dispersed into the ISM by the explosion). Of the weak $s$-process elements listed above, the easiest to measure in stellar spectra is $\mathrm{Sr}$.

Therefore, we will calculate the Galactic evolution of the abundance of ${ }^{40} \mathrm{~K}$ relative to that of Sr. As we did for Th and U, we begin by deriving the relation between the stable reference element and Fe. Employing the observations of Gratton and Sneden (1994), we derive:

$$
[S r / F e]=(-0.18 \pm 0.04)-(0.29 \pm 0.06)[F e / H]
$$

from 11 stars with $[\mathrm{Fe} / \mathrm{H}]>-1.3$. Notably, the slope of this relation is nearly identical to that of the $[\mathrm{Eu} / \mathrm{Fe}]$ trend (Eq. 2), implying that $\mathrm{Sr}$ and $\mathrm{Eu}$ come from the same sources. However, being a secondary element, one would expect Sr to have a different dependence on Fe. The close agreement between the two slopes is probably fortuitous. As above, we make use of Clayton's equations to derive the evolution of the ${ }^{40} \mathrm{~K} / \mathrm{Sr}$ ratio. We adopt the value of the half-life for ${ }^{40} \mathrm{~K}$ given by Fowler (1990), 1.25 Gyr, which includes the effects of the branched decay of this isotope. Unfortunately, the production ratio of ${ }^{40} \mathrm{~K} / \mathrm{Sr}\left(\equiv\left({ }^{40} \mathrm{~K} / \mathrm{Sr}\right)_{0}\right)$ is not well known, so, we have set its value to give the present Solar System abundance ratio; we get $\left({ }^{40} \mathrm{~K} / \mathrm{Sr}\right)_{0}=0.64$. The resulting trend of $\left[{ }^{40} \mathrm{~K} / \mathrm{Fe}\right]$ is shown in Fig. 5. The curve does not pass through zero at $\mathrm{t}=10.5 \mathrm{Gyr}$, because the Sun has a greater $\mathrm{Sr} / \mathrm{Fe}$ ratio than other solar metallicity stars used to derive Eq. 5; this might be another example of an 
anomalous solar parameter value related to habitability (see Gonzalez 1999b).

Even before we produced Fig. 5, it was already clear that $\left[{ }^{40} \mathrm{~K} / \mathrm{Fe}\right]$ should decline with time given the observed decline of $[\mathrm{O} / \mathrm{Fe}]$ with time. This is the case because $\mathrm{O}$ is injected

into the ISM by the same types of stars as is ${ }^{40} \mathrm{~K}$. However, this does not mean that ${ }^{40} \mathrm{~K}$ is declining in the ISM, on the contrary, its mass fraction is increasing (Podosek et al. 1999).

Finally, we should note that our calculations of the time evolution of long-lived radioisotopes assume that the evolution of their abundances can be approximated by smooth functions. This assumption is justified if the immediately preceding SNe in the vicinity of the forming planetary system contributed only a relatively small fraction of freshly synthesized isotopes to it. Podosek et al. (1999) estimate that about $3 \%$ of the ${ }^{40} \mathrm{~K}$ in the early Solar Nebula was contributed by a massive star SNe immediately preceding its formation. This is sufficiently small not to invalidate our assumption.

\subsection{Evolution of Terrestrial Planet Radiogenic Heating}

Now that we know the evolution of the abundances of the long-lived radionuclides in the ISM, we can estimate the evolution of the radiogenic heating in a terrestrial planet. We will use the Earth as the standard in the following discussion for obvious reasons.

In the calculations of the previous section, we adopted what are believed to be the isotopic abundances characteristic of the Solar System as a whole prior to the fractionation of the volatile elements. However, the present bulk compositions of the planets vary considerably. In the Earth, the noble elements (He, Ne, Ar, etc.) and other volatiles (H, CNO) are greatly under-represented relative to the solar photospheric abundances. Kargel and Lewis (1993) give estimates for the amounts of $\mathrm{K}$, Th, and $\mathrm{U}$ in the core and the mantle+crust of the Earth; they estimate 210 ppm by weight of $\mathrm{K}$ and no significant $\mathrm{U}$ 
or Th in the core. More recently, Lodders (1995) and Kargel (1995) argue for a higher core abundance of K (550 ppm), based on the assumption that the Earth accreted from material similar in composition to enstatite chondrites. We will adopt this second estimate for the core abundance of K and Kargel and Lewis's (1993) values for the mantle+crust abundances of K, Th, and U. Herndon (1993) estimate the mass of U in the core based on the composition of the Abee enstatite meteorite; he quotes masses of $5.8 \times 10^{16} \mathrm{~g}$ for ${ }^{235} \mathrm{U}$ and $8.0 \times 10^{18} \mathrm{~g}$ for ${ }^{238} \mathrm{U}$ in the core, both of which we adopt. To calculate the mass of ${ }^{232} \mathrm{Th}$ in the core, we assume the same ratio of Th/U as quoted by Anders and Grevesse (1999) for meteorites. This set of abundances results in bulk-Earth-to-C1-chondrite ratios of: $\mathrm{U}=1.92 ; \mathrm{Th}=1.89 ; \mathrm{K}=0.62$. Relative to $\mathrm{Fe}$, these ratios are: $\mathrm{U} / \mathrm{Fe}=1.12 ; \mathrm{Th} / \mathrm{Fe}$ $=1.10 ; \mathrm{K} / \mathrm{Fe}=0.36$.

While we adopt specific values for the abundances of the radioactive isotopes in this section, we need to be clear that some of these numbers are not yet well known. This is particularly the case for $\mathrm{K}$, for which the core abundance is still controversial, and for which the degree of fractionation during planet formation is also uncertain. Therefore, our quoting numbers to two significant digits does not imply that the estimates are to be accepted at that level of precision.

In order to calculate the radioactive heating, we also need to know the amount of energy produced per decay; which we adopt from Faure (1977). We show the results of our calculations for the Earth in Fig. 6. Our calculations give a present-day radiogenic heat production rate of $3.25 \times 10^{20} \mathrm{erg} \mathrm{s}^{-1}$ for the entire Earth; the core contributes $22 \%$. The observed present global heat loss rate at the Earth's surface is $(4.42 \pm 0.10) \times 10^{20}$ $\operatorname{erg~s}^{-1}$ (Pollack et al. 1993). It is not clear if the deficit can be accounted for entirely from uncertainties in the radioisotope abundances. More likely, other heat sources, such as secular cooling of the core and mantle, can make up the difference (see Breuer and Spohn 
1993 and references cited therein).

Combining the results of this section with those of the previous one, we can calculate the evolution of the radiogenic heat production in a terrestrial planet formed at any time since the formation of the Milky Way. There are two ways of approaching this problem: 1) we can keep the planet mass constant, or 2) we can assume that the planet mass scales with $10^{1.5[\mathrm{Fe} / \mathrm{H}]}$ (see Section 2.1). The first case is applicable to a discussion that assumes that a terrestrial planet near the Earth's mass is a requirement for complex life. The second case is probably closer to reality, but it complicates the interpretation, since we are introducing yet another parameter.

As already noted, the bulk composition of the Earth differs from C1-chondrites, whose abundance pattern is a close match to that of the solar photosphere (except for the most volatile elements). The differences are most likely due to the higher nebular temperatures in the inner protoplanetary disk, where the Earth formed. The place of formation of the C1-chondites allowed them to experience relatively less volatile fractionation during their condensation. Therefore, in order to calculate the radiogenic heating in the interior of an Earth-like terrestrial planet, we must first scale the stellar K/Fe, Th/Fe, and U/Fe abundance ratios from Fig. 5 using the bulk Earth-to-C1-chondrite ratios given above. We show the results of such a calculation in Fig. 7. They indicate that a terrestrial planet with the same formation time and age as the Earth produces only $36 \%$ as much radiogenic heat, and one forming today will, in $4.5 \mathrm{Gyr}$, produce only $21 \%$ as much. This is due to the smaller value of ${ }^{40} \mathrm{~K} / \mathrm{Fe}$ predicted by our Galactic chemical evolution calculations. It is not clear if the ${ }^{40} \mathrm{~K} / \mathrm{Fe}$ ratio in the Earth really is anomalously high for its formation time, so we have also calculated the radiogenic heating in an Earth-mass terrestrial planet assuming the Earth's current radiogenic heat production is typical (Fig. 7, dotted curve). In this second case, an Earth-twin formed today will, in 4.5 Gyr, have $60 \%$ of the present Earth's 
radiogenic heat production.

The radiogenic heat production for variable planet mass is shown in Fig. 8. As can be seen in the figure, for a terrestrial planet with a mass that scales with $10^{1.5[\mathrm{Fe} / \mathrm{H}]}$, the radiogenic heat production levels off. However, if we are correct in our speculation that significantly higher metallicities lead to less habitable systems (discussed in Section 2), then larger terrestrial planets formed in the future at our distance from the Galactic center will not necessarily be as habitable as the present Earth.

\section{OTHER GALAXIES}

Like stars, galaxies span a very broad range in luminosity (and mass). The Milky Way is classified as a large spiral galaxy of morphological type $\mathrm{SAB}(\mathrm{rs}) \mathrm{bc}$ with an absolute blue magnitude, $\mathrm{M}_{\mathrm{B}}$, of $-20.2 \pm 0.15$ magnitudes (de Vaucouleurs 1982). It is one of three spirals in the Local Group.

It is now well established from observations that a metallicity-luminosity correlation exists among galaxies of all types. Pilyugin and Ferrini (2000) discuss this correlation among late-type galaxies (see their Fig. 1); the sense of the correlation is such that luminous galaxies are more metal-rich than less luminous ones. We can calculate the fraction of galaxies that are less luminous than the Milky Way (and therefore more metal-poor) by comparing the Milky Way's luminosity to the nearby galaxy luminosity function. The observed luminosity function of a sample of nearby galaxies is usually fitted to a Schechter function (Schechter 1976), which is of the form:

$$
\phi(L) d L=\phi^{\star}\left(\frac{L}{L^{\star}}\right)^{\alpha} \exp \left[-\frac{L}{L^{\star}}\right] \frac{d L}{L^{\star}}
$$

where 


$$
\frac{L}{L^{\star}}=10^{0.4\left(M^{\star}-M\right)}
$$

Folkes et al. (1999) present results of an analysis of a preliminary sample of 5869 galaxy spectra from the $2 \mathrm{dF}$ Galaxy Redshift Survey. A Schechter function fit to their dataset yields the following values for the constants in Eqs. 6 and 7 (in blue light): $\mathrm{M}^{\star}=-19.73$ magnitudes and $\alpha=-1.28$. Restricting our calculations to $\mathrm{M}_{\mathrm{B}}$ between -14 and -22 magnitudes, we find: 1) the Milky Way is among the $0.7 \%$ most luminous galaxies in the blue, and 2) these most luminous galaxies contain $16 \%$ of the blue light (and, presumably, $16 \%$ of the stars). Of course, this is only an approximate estimate; we did not include in our calculation the cosmic dispersion in metallicity at a given luminosity nor the spread in metallicity in a given galaxy. Exclusion of these other factors may increase the uncertainty in our estimate by a few percent.

If we were to assume that the total number of stars in a galaxy is proportional to its luminosity in the blue, then we could just equate the relative luminosities given above to the relative numbers of stars. Unfortunately, in the blue we are sensitive to the relatively small number of massive blue stars recently formed in a galaxy, so we are in effect biased towards galaxies with active star formation activity. To reduce this bias, we can repeat the above calculation with observations obtained with a redder bandpass. Reed (2000) estimates that massive stars contribute about $2 \%$ of the Milky Way's luminosity in the visual band. De Vaucouleurs (1982) gives the Milky Way's $\mathrm{M}_{\mathrm{V}}$ as $-20.73 \pm 0.16$ magnitudes. Blanton et al. (2000) derive luminosity functions in five bandpasses from Sloan Digital Sky Survey commissioning data. None of the Sloan filters is equivalent to the Johnson $\mathrm{V}$ filter, but the average wavelength of the Sloan $g^{\star}$ and $r^{\star}$ filters is equivalent to it. The Schechter function constants corresponding to the average of the Sloan $\mathrm{g}^{\star}$ and $\mathrm{r}^{\star}$ filters are: $\mathrm{M}^{\star}=-20.40$ magnitudes and $\alpha=-1.23$. Restricting our calculations to $\mathrm{M}_{\mathrm{V}}$ between -15 and -23 
magnitudes, we find: 1) the Milky Way is among the $1.3 \%$ most luminous galaxies in the visual, and 2) these most luminous galaxies contain $23 \%$ of the visual light.

Of course, these results do not apply to the distant (and hence early) universe (e.g., the Hubble Deep Fields). The metallicity was much less then (see Wasserburg and Qian 2000 for a discussion of the very early metallicity evolution of the universe). Also, the radiation environment was much more hostile due to the higher frequency of transient radiation events (especially in the center of a galaxy, where the metallicity is highest). Therefore, it is likely that the Hubble Deep Fields are completely devoid of habitable planets (except possibly for a few nearby foreground galaxies). Until recently, studies of the evolution of star formation activity in the larger universe indicated that it peaked near a redshift of 2, which corresponds to about 3 Gyrs after the Big Bang (Blain and Natarajan 2000). However, more recently, some have begun to argue that the star formation rate peaked prior to a redshift of 2 (e.g., Metcalfe et al. 2000).

\section{SUMMARY}

We have argued that there exists a zone of enhanced habitability in the Milky Way, which we have termed the Galactic Habitable Zone (GHZ). One of the more important factors is the metallicity of the interstellar matter out of which a planetary system forms. This determines the masses of the terrestrial planets in the system (and probably also the gas giants). This is based on the assumption that terrestrial planet mass scales with the surface density of solids in a protoplanetary disk, such that lower metallicity leads to smaller planets. We estimate, very approximately, that a metallicity at least half that of the Sun is required to build a habitable terrestrial planet. These assumptions need to be

tested with simulations of planetary formation with adjustable initial metallicity starting with the collapse of the birth cloud. 
Also important is the decrease in the interstellar medium abundances of the long-lived radioisotopes, ${ }^{40} \mathrm{~K},{ }^{235,238} \mathrm{U}$, and ${ }^{232} \mathrm{Th}$ relative to Fe. Given the lack of a suitable alternative geothermal energy source, radiogenic heating is a necessary requirement for the long-term maintenance of a terrestrial planet's habitability via climate stability provided by the carbon cycle. Of somewhat lesser importance for habitability are the ratios: $\mathrm{C} / \mathrm{O}, \mathrm{Si} / \mathrm{Fe}$, $\mathrm{Mg} / \mathrm{Fe}$, and $\mathrm{S} / \mathrm{Fe}$. These affect the water content, the core to mantle mass ratio, and the state of the core of a terrestrial planet. The least certain aspect of our calculations concerns the abundance of ${ }^{40} \mathrm{~K}$, both in the interstellar medium and in terrestrial planets. Additional research on all aspects of ${ }^{40} \mathrm{~K}$ abundances is warranted.

In the process of defining the GHZ, we found it necessary first to establish the links between the Circumstellar Habitable Zone (CHZ) and Galactic scale phenomena. As a result of our review of studies of the $\mathrm{CHZ}$, we found that many important factors are not included in its general definition. These include the radial composition gradient in the early protoplanetary disk (especially with regard to ${ }^{40} \mathrm{~K}$ ), the radial dependence of impactor energy, and details of the late stages of planet accretion.

Based on Galactic chemical evolution alone, we find, not surprisingly, that the thin disk near the Sun is the most likely place for Earth-like planets to form in the present time. On average, the inner disk should contain terrestrial planets larger than the Earth, and the outer disk is likely to contain smaller terrestrial planets. Given recent evidence of a metallicity dependence on giant planet formation, they should also be more common in the inner Galactic disk. The bulge should contain many Earth-mass planets but relatively few Earth-like planets, given the different mix of elements among its stars. The evolving concentration of the geophysically important radioisotopes in the ISM establishes a window of time (albeit with fuzzy boundaries) in the history of the Milky Way during which terrestrial habitable planets with long-lasting geological activity can exist. That window is 
slowly closing with a timescale of billions of years.

Observations of other galaxies show that the Milky Way's star formation history is probably not atypical. Star formation in other large galaxies probably peaked at about the same time as in the Milky Way. The observed correlation in the nearby universe between the metallicity of a galaxy and its luminosity implies that low luminosity galaxies are unlikely to contain Earth-mass planets yet.

Of course, the definition of the GHZ presented in this study is not complete, as we did not discuss Galactic scale constraints not directly related to Galactic chemical evolution. These include threats from transient radiation events and dynamical perturbations of comet and planet orbits (to be discussed in future papers in this series). Consideration of these other constraints lead us to exclude the bulge and inner disk from the GHZ. To summarize, then, the GHZ is an annulus in the thin disk of the Milky Way that migrates outward with time as the heavy elements build up.

The authors thank J. J. Cowan, J. Lissauer, and G. Wallerstein for helpful advice and comments. The constructive and thoughtful critiques of the reviewers, J. Lissauer, C. P. McKay, and V. Trimble, helped to clarify the presentation and are greatly appreciated. GG acknowledges support from the Kennilworth Fund of the New York Community Trust. 


\section{REFERENCES}

Anders, E., and N. Grevesse 1989. Abundances of the elements: meteoritic and solar. Geochim. et Cosmochem. Acta 53, 197-214.

Balazs, B. S. 1988. The Galactic belt of intelligent life. In Bioastronomy - The next steps (G. Max, Ed.), pp. 61-66. Kluwer Academic Pub., Dordrecht.

Blain, A. W., and P. Natarajan 2000. Gamma-ray bursts and the history of star formation. MNRAS 312, L35-38.

Blanton, M. R., et al. 2001. The luminosity function of galaxies in SDSS commissioning data. AJ, submitted.

Brakenridge, G. R. 1981. Terrestrial paleoenvironmental effects of a late quaternary-age supernova. Icarus 46, 81-93.

Breuer, D., and T. Spohn 1993. Cooling of the Earth, Urey ratios, and the problem of potassium in the core. Geophys. Res. Let. 20, 1655-1658.

Buffett, B. A. 2000. Earth's core and the geodynamo. Science 288, 2007-2012.

Butler, R. P. et al. 2000. Statistical properties of extrasolar planets. In Planetary systems in the universe: Observation, formation, and evolution (Penny A, Artymowicz P, Lagrange A -M, and Russell, Eds.), ASP Conference Series, in press.

Butler, R. P. et al. 2001. Two new planets from the Anglo-Australian planet search. AJ, submitted.

Carigi, L. 1996. Models of Chemical Evolution of the Galactic Disk: Considering Different SFR, IMF, and Infall Rates. Rev. Mexicana Astron. Astrofis. 32, 179-192. 
Chen, Y. Q., P. E. Nissen, G. Zhao, H. W. Zhang, and T. Benoni 2000. Chemical composition of $90 \mathrm{~F}$ and $\mathrm{G}$ dwarfs. A\&AS 141, 491-506.

Clarke, J. N. 1981. Extraterrestrial intelligence and galactic nuclear activity. Icarus 46, 94-96.

Clayton, D. D. 1988. Nuclear cosmochronology within analytic models of the chemical evolution of the solar neighborhood. MNRAS 234, 1-36.

Cowan, J. J. 1999, private communication.

Cowan, J. J., A. McWilliam, C. Sneden, and D. L. Burris 1997. The thorium chronometer in CS 22892-052: estimates of the age of the Galaxy. ApJ 480, 246-254.

Cowan, J. J., B. Pfeiffer, K.-L. Kratz, F.-K. Thielemann, C. Sneden, S. Burles, D. Tytler, and T. Beers 1999. R-process abundances and chronometers in metal-poor stars. ApJ 521, 194-205.

de Vaucouleurs, G. 1982. Five crucial tests of the cosmic distance scale using the Galaxy as fundamental standard. Proc. Astron. Soc. Aus. 4(4), 320-327.

Draganic, I. G., E. Bjergbakke, Z. D. Draganic, and K. Sehested 1991. Decomposition of ocean waters by potassium-40 radiation 3800 Ma ago as a source of oxygen and oxidizing species. Precam. Res. 52, 337-345.

Edvardsson, B., J. Andersen, B. Gustafsson, D. L. Lambert, P. E. Nissen, and J. Tomkin 1993. The chemical evolution of the Galactic disk: I. analysis and results. A\&A 275, 101-152.

Ellis, J. and D. N. Schramm 1995. Could a nearby supernova explosion have caused a mass extinction? Proc. Nat. Acad. Sci. 92, 235-238. 
Faure, G. 1977. Principles of Isotope Geology, John Wiley \& Sons, New York.

Folkes, S. et al. 1999. The 2dF Galaxy redshift survey: spectral types and luminosity functions. MNRAS 308, 459-472.

Fowler, C. M. R. 1990. The Solid Earth: An Introduction to Global Geophysics, Cambridge Univ. Press, Cambridge.

Franck, S. 1998. Evolution of the global mean heat flow over 4.6 Gyr. Tectonophysics 291, $9-18$.

Franck, S., A. Block, W. von Bloh, C. Bounama, H. J. Schellnhuber, and Y. Svirezhev 2000a. Reduction of biosphere life span as a consequence of geodynamics. Tellus 52B, 94-107.

Franck, S., A. Block, W. von Bloh, C. Bounama, M. Steffen, D. Schonberner, and H. J. Schellnhuber 2000b. Determination of habitable zones in extrasolar planetary systems: where are Gaia's sisters? JGR 105, 1651-1658.

Franck, S., K. Kossacki, and C. Bounama 1999. Modelling the global carbon cycle for the past and future evolution of the Earth system. Chem. Geol. 159, 305-317.

Gaidos, E. J. 2000. A cosmochemical determinism in the formation of Earth-like planets. Icarus 145, 637-640.

Gaidos, E. J., and G. Gonzalez 2000. Young solar analogs. in preparation.

Garnett, D. R., et al. 1999. Carbon in Spiral Galaxies from Hubble Space Telescope Spectroscopy. ApJ 513, 168-179.

Gilliland, R. L. et al. 2000. A lack of planets in 47 Tucanae from an HST search. ApJ 545, L47-51. 
Gonzalez, G. 1999a. Are stars with planets anomalous? MNRAS 308, 447-458.

Gonzalez, G. 1999b. Is the sun anomalous? Astron. \& Geophys. 40, 5.25-5.29.

Gonzalez, G., C. Laws, S. Tyagi, and B. E. Reddy 2001. Parent stars of extrasolar planets VI: Abundance analyses of 20 new systems. AJ 121, 432-452.

Goswami, A., and N. Prantzos 2000. Abundance evolution of intermediate mass elements (C to Zn) in the Milky Way halo and disk. A\&A 359, 191-212.

Gratton, R. G., and C. Sneden 1994. Abundances of neutron-capture elements in metal-poor stars. A\&A 287, 927-946.

Gratton, R. G., E. Carretta, F. Matteucci, and C. Sneden 2000. Abundances of light elements in metal-poor stars. IV. $[\mathrm{Fe} / \mathrm{O}]$ and $[\mathrm{Fe} / \mathrm{Mg}]$ ratios and the history of star formation in the solar neighborhood. A\&A 358, 671-681.

Greenberg, R., P. Geissler, B. R. Tufts, and G. Hoppa 2000. Habitability of Europa's crust: the role of tidal-tectonic processes. J. Geophys. Res. 105, 17,551-17,562.

Hart, M. H. 1979. Habitable zones about main sequence stars. Icarus 37, 351-357.

Henry, R. B. C., and G. Worthey 1999. The distribution heavy elements in spiral and elliptical galaxies. PASP 111, 919-945.

Henry, T. J. 1998. Suspicious Characters Lurking in the Solar Neighborhood. In Brown Dwarfs and Extrasolar Planets (Rebolo R, Martin E L, Osorio M R Z, Eds.), pp. 28-35. Book Crafters, San Francisco.

Henry, T. J. 1999. private communication.

Herndon, J. M. 1993. Feasibility of a nuclear fission reactor at the center of the Earth as the energy source of the geomagnetic field. J. Geomag. Geoelectric. 45, 423-437. 
Hou, J. L, N. Prantzos, and S. Boissier 2000. Abundance gradients and their evolution in the Milky Way disk. A\&A 362, 921-936.

Huang, S.-S. 1959. Occurrence of life in the universe. American Scientist 47, 397-402.

Kappeler, F., H. Beer, and K. Wisshak 1989. s-process nucleosynthesis - nuclear physics and the classical model. Rep. Prog. Phys. 52, 945-1013.

Karato, S.-I., and H. Jung 1998. Water, partial melting and the origin of the seismic low velocity and high attenuation zone in the upper mantle. Earth Plan. Sci. Lett. 157, 193-207.

Kargel, J. S. 1995. A possible enstatite meteorite-earth connection and potassium in Earth's core. Meteoritics 30, 5-7.

Kargel, J. S., and J. S. Lewis 1993. The composition and early evolution of Earth. Icarus 105, $1-25$.

Kasting, J. F., D.P. Whitmire, R.T. Reynolds 1993. Habitable zones around main sequence stars. Icarus 101, 108-128.

Lewis, J. R., and K. C. Freeman 1989. Kinematics and chemical properties of the old disk of the galaxy. AJ 97, 139-162.

Lewis, J. S. 1997. Physics and Chemistry of the Solar System. Academic Press, San Diego, CA.

Lewis, J. S. 1998. Worlds Without End: the exploration of planets known and unknown. Perseus Books, Reading, MA.

Lineweaver, C. H. 2000. An estimate of the age distribution of terrestrial planets in the universe: quantifying metallicity as a selection effect. Icarus, in press. 
Lissauer, J. J. 1995. Urey prize lecture: on the diversity of plausible planetary systems. Icarus 114, 217-236.

Lodders, K. 1995. Alkali elements in the Earth's core: evidence from enstatite meteorites. Meteoritics 30, 93-101.

Lodders, K. and B. Fegley, Jr. 1998. The Planetary Scientist's Companion. Oxford University Press, New York.

Marochnik, L. S. 1984. On the position of the Sun in the Galaxy. Astrophysics 19, 278-283.

Mathews, G. J., G. Bazan, and J. J. Cowan 1992. Evolution of heavy-element abundances as a constraint on sites for neutron-capture nucleosynthesis. ApJ 391, 719-735.

McKay, C. P. 1996. Time for intelligence on other planets. In Circumstellar Habitable Zones: proceedings of the first international conference (Doyle L R, Ed.), pp. 405-419. Travis House Publications, Menlo Park, CA.

McWilliam, A. 1997. Abundance ratios and Galactic chemical evolution. ARA\&A 35, $503-556$.

Metcalfe, N., T. Shanks, A. Campos, H. J. McCracken, and R. Fong 2000. Galaxy number counts - V. Ultra-deep counts: The Herschel and Hubble Deep Fields. MNRAS, submitted.

Molla, M., F. Ferrini, and G. Gozzi 2000. Galactic bulges. MNRAS 316, 345-356.

Norris, J. E. 1999. The Chemical Abundance Structure of the Galactic Halo. In The third Stromlo Symposium: The Galactic Halo (Gibson B K, Axelrod T S, and Putnam M E, Eds.), pp. 213-224. Book Crafters, San Francisco.

Moulton, K. L., and R. A. Berner 1998. Quantification of the effect of plants on weathering; studies in Iceland. Geology 26(10), 895-898. 
Pagel, B. 1997. Nucleosynthesis and Galactic Chemical Evolution. Cambridge University Press, Cambridge.

Pagel, B. E. J., and G. Tautvaisiene 1995. Chemical evolution of primary elements in the Galactic disk: an analytical model. MNRAS 276, 505-514.

Pilyugin, L. S., and F. Ferrini 2000. On the origin of the luminosity-metallicity relation for late-type galaxies: spirals to irregulars transition. A\&A 358, 72-76.

Podosek, F. A., R. H. Nichols, Jr., J. C. Brandon, B. S. Meyer, U. Ott, C. L. Jennings, and N. Luo 1999. Potassium, stardust, and the last supernova. Geochimica et Cosmochemica Acta 63, 2351-2362.

Pollack, H. N., S. J. Hurter, J. R. Johnson 1993. Heat flow from the Earth's interior analysis of the global data set. Rev. Geophys. 31(3), 267-280.

Portinari, L., C. Chiosi, and A. Bressan 1998. Galactic chemical enrichment with new metallicity dependent stellar yields. A\&A 334, 505-539.

Pratzos, N., M. Hashimoto, and K. Nomoto 1990. The s-process in massive stars: yields as a function of stellar mass and metallicity. A\&A 234, 211-229.

Prochaska, J. X., S. O. Naumov, B. W. Carney, A. McWilliam, and A. M. Wolfe 2000. The Galactic thick disk stellar abundances. AJ 120, 2513-2549.

Reed, B. C. 2000. New estimates of the scale height and surface density of OB stars in the solar neighborhood. AJ 120, 314-318.

Rich, R. M., and A. McWilliam 2000. Abundances of stars in the galactic bulge obtained using the Keck Telescope. Proc. SPIE 4005, 150-161.

Rocha-Pinto, H. J. and W. J. Maciel 1996. The metallicity distribution of G dwarfs in the solar neighborhood. MNRAS 279, 447-458. 
Rocha-Pinto, H. J., W. J. Maciel, J. Scalo, and C. Flynn 2000. Chemical enrichment and star formation in the Milky Way. I. Sample description and chromospheric age-metallicity relation. A\&A 358, 850-868.

Rolleston, W. R. J., S. J. Smartt, P. L. Dufton, and R. S. I. Ryans 2000. The Galactic metallicity gradient. A\&A 363, 537-554.

Sagan, C., and J. S. Shklovsky 1966. Intelligent life in the universe. Holden-Day, San Francisco.

Samland, M. 1998. Modeling the evolution of disk galaxies. II. Yields of massive stars. ApJ 496, 155-171.

Schechter, P. L. 1976. An analytic expression for the luminosity function of galaxies. ApJ 203, 297-306.

Schenk, P. M., E. Asphaug, W. B. McKinnon, H. J. Melosh, and P. R. Weissman 1996. Cometary nuclei and tidal disruption: the geologic record of crater chains on Callisto and Ganymede. Icarus 121, 249-274.

Tackley, P. J. 2000. Mantle convection and plate tectonics: toward an integrated physical and chemical theory. Science 288, 2002-2007.

Thielemann, F.-K., K. Nomoto, and M. Hashimoto 1996. Core-collapse supernovae and their ejecta. ApJ 460, 408-436.

Timmes, F. X., S. E. Woosley, and T. A. Weaver 1995. Galactic chemical evolution: Hydrogen through Zinc. ApJS 98, 617-658.

Trimble, V. 1997a. Origin of the biologically important elements.Origins of Life Evol. Biosphere 27, 3-21. 
Trimble, V. 1997b. Galactic chemical evolution: implications for the existence of habitable planets. In Extraterrestrials - Where are They? (Zuckerman B. \& Hart M., Eds.), pp. 184-191. Cambridge University Press, Cambridge.

Tucker, W. C. 1981. Astrophysical crises in the evolution of life in the Galaxy. In Life in the Universe (Billingham J., Ed.), pp. 287-296. The MIT Press, Cambridge.

Ward, P., and D. Brownlee 2000. Rare Earth. Copernicus, New York.

Wasserburg, G. J., and Y.-Z. Qian 2000. A model of metallicity evolution in the early universe. ApJ 538, L99-L102.

Weidenschilling, S. J., and F. Marzari 1996. Gravitational scattering as a possible origin for giant planets at small distances. Nature 384, 619-621.

Wetherill, G. W. 1996. The formation and habitability of extra-solar planets. Icarus 119, 219-238.

Whitmire, D. P. and R. T. Reynolds 1996. Circumstellar habitable zones: astronomical considerations. In Circumstellar Habitable Zones (L. R. Doyle, Ed.), pp. 117-142. Menlo Park, Travis House Publications.

Whittet, D. 1997. Astronomy \& Geophys. 38(5), 8.

Williams, D. M., J. F. Kasting, and R. A. Wade 1997. Habitable moons around extrasolar giant planets. Nature 385, 234-236.

Woolf, V. M., J. Tomkin, and D. L. Lambert 1995. The r-process element Europium in Galactic disk F and G dwarf stars. ApJ 453, 660-672. 
Fig. 1. - Terrestrial planet mass is plotted against time since the formation of the Milky Way for the ISM in the solar neighborhood (diagram a). The nominal trend calculated from Eq. 1 is shown as a solid curve; mass of planet is assumed proportional to $10^{1.5[\mathrm{Fe} / \mathrm{H}]}$ (see Section 2.1). The one sigma upper and lower bounds are shown as dotted curves. The Sun is shown as an open circle. Terrestrial planet mass is plotted against Galactocentric distance for the present ISM (diagram b).

Fig. 2.- Locus of points on the time-Galactocentric distance plane corresponding to solar metallicity of the ISM. The diagram is divided into metal-poor and metal-rich regions (with respect to solar metallicity). Symbol meanings are the same as in Fig. 1.

Fig. 3.- Trend of $[\mathrm{Eu} / \mathrm{Fe}]$ with $[\mathrm{Fe} / \mathrm{H}]$ using data from Woolf et al. (1995), corrected for $\mathrm{R}_{\mathrm{m}}=8.8 \mathrm{kpc}$ (dots; panel $\mathbf{a}$ ); the bracket notation employed here and in the following captions is a logarithmic abundance scale relative to the Sun (see text for a definition). The data have been separated into three groups in panel $\mathbf{b}$; the standard error of the mean is shown for each point. Equation 2 is shown as a line in panel $\mathbf{b}$.

Fig. 4. - The present relative abundances of the $r$-process radioisotopes, as $\left[{ }^{232} \mathrm{Th},{ }^{235} \mathrm{U}\right.$, $\left.{ }^{238} \mathrm{U} / \mathrm{Eu}\right]$, as a function of a star's formation time, t. The abundances shown are relative to the present meteoritic abundances from Anders and Grevesse (1989). The Solar System value is indicated with an open circle.

Fig. 5.- The $\left[{ }^{232} \mathrm{Th},{ }^{235} \mathrm{U},{ }^{238} \mathrm{U},{ }^{40} \mathrm{~K} / \mathrm{Fe}\right]$ values in a star $4.5 \mathrm{Gyr}$ after its formation as a function of formation time, t. The error bars shown at the time of the formation of the solar system are based on an uncertainty of $\pm 10 \%$ in the production ratios. The solar system value is indicated with an open circle.

Fig. 6.- Evolution of radiogenic heating from the decay of ${ }^{40} \mathrm{~K},{ }^{232} \mathrm{Th},{ }^{235} \mathrm{U}$, and ${ }^{238} \mathrm{U}$ in the Earth's interior. The dot represents the observed present heat loss at the surface of the 
Earth (Pollack et al. 1993). Here t corresponds to the time since the formation of the Earth.

Fig. 7.- Radiogenic heating in an Earth-mass planet 4.5 Gyr after its formation relative to the present radiogenic heating in the Earth as a function of formation time (solid curve). This is based on the data in Figs. 5 and 6 . The data have also been multiplied by a constant in order to pass through unity at $\mathrm{t}=10.5$ Gyr (dotted curve).

Fig. 8. - Same as Fig. 7 but for planet mass that is proportional to $10^{1.5[\mathrm{Fe} / \mathrm{H}]}$; the solid and dotted curves have the same meaning as before. The planet mass relative to the Earth is indicated by a dashed curve. 

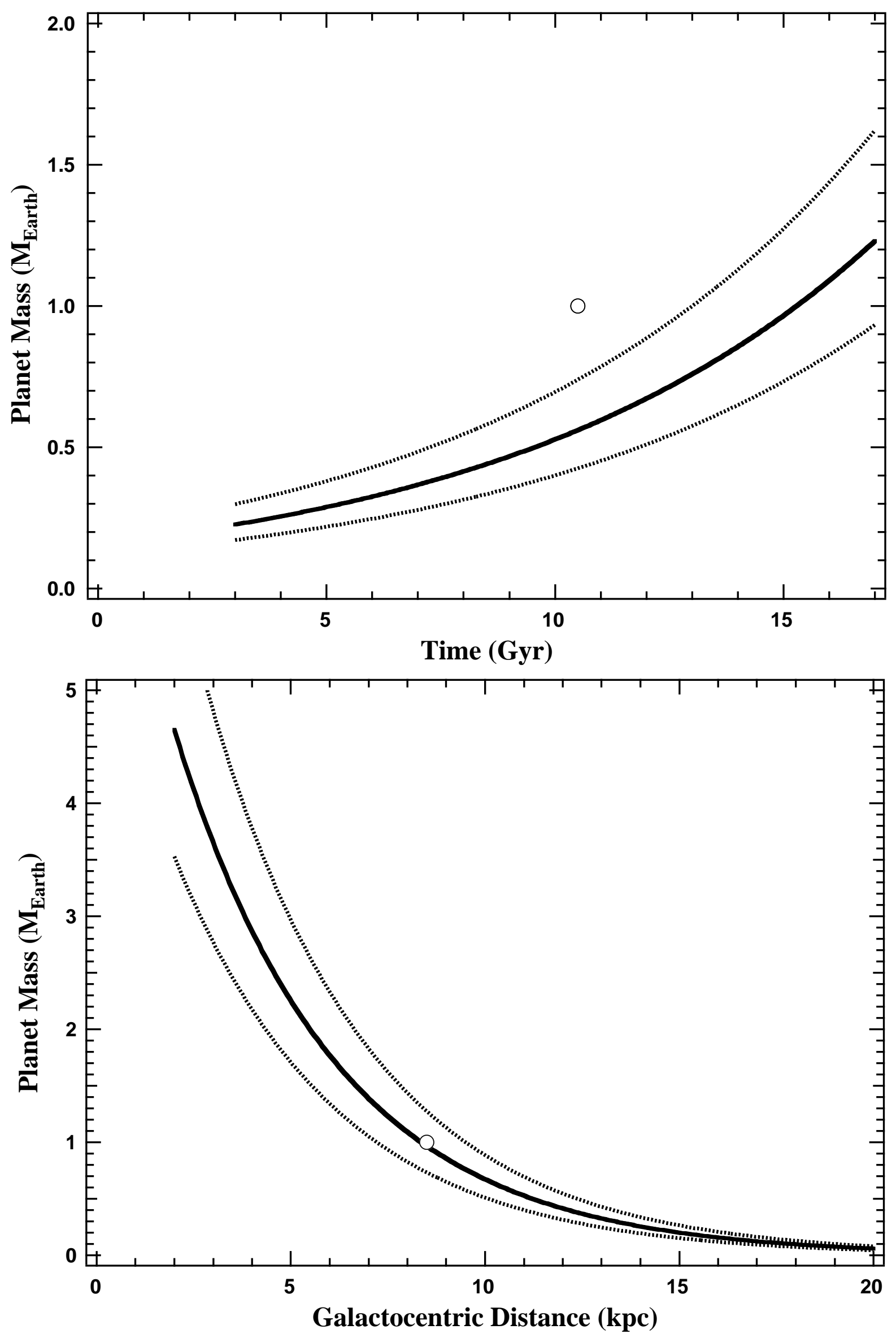


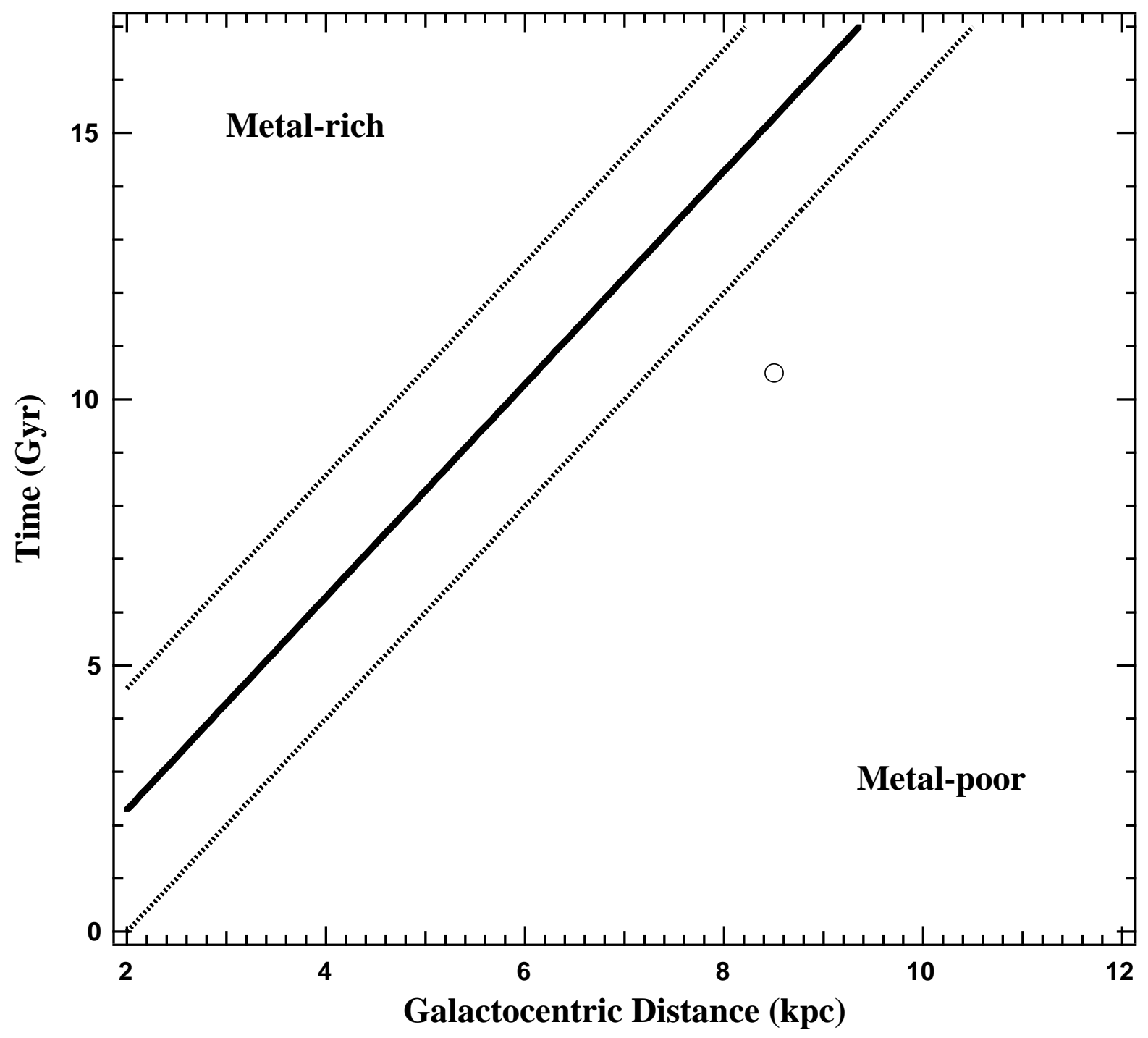



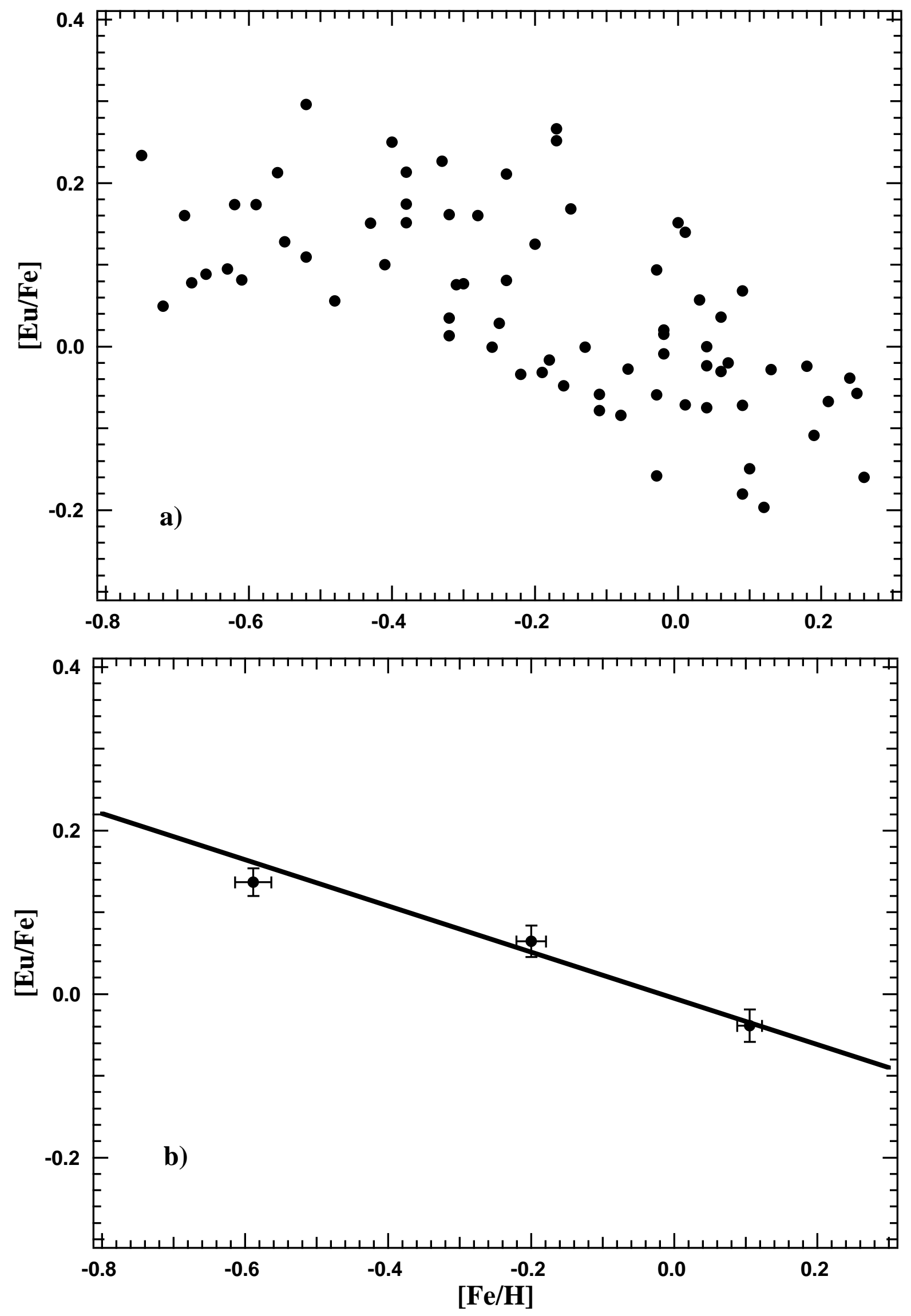


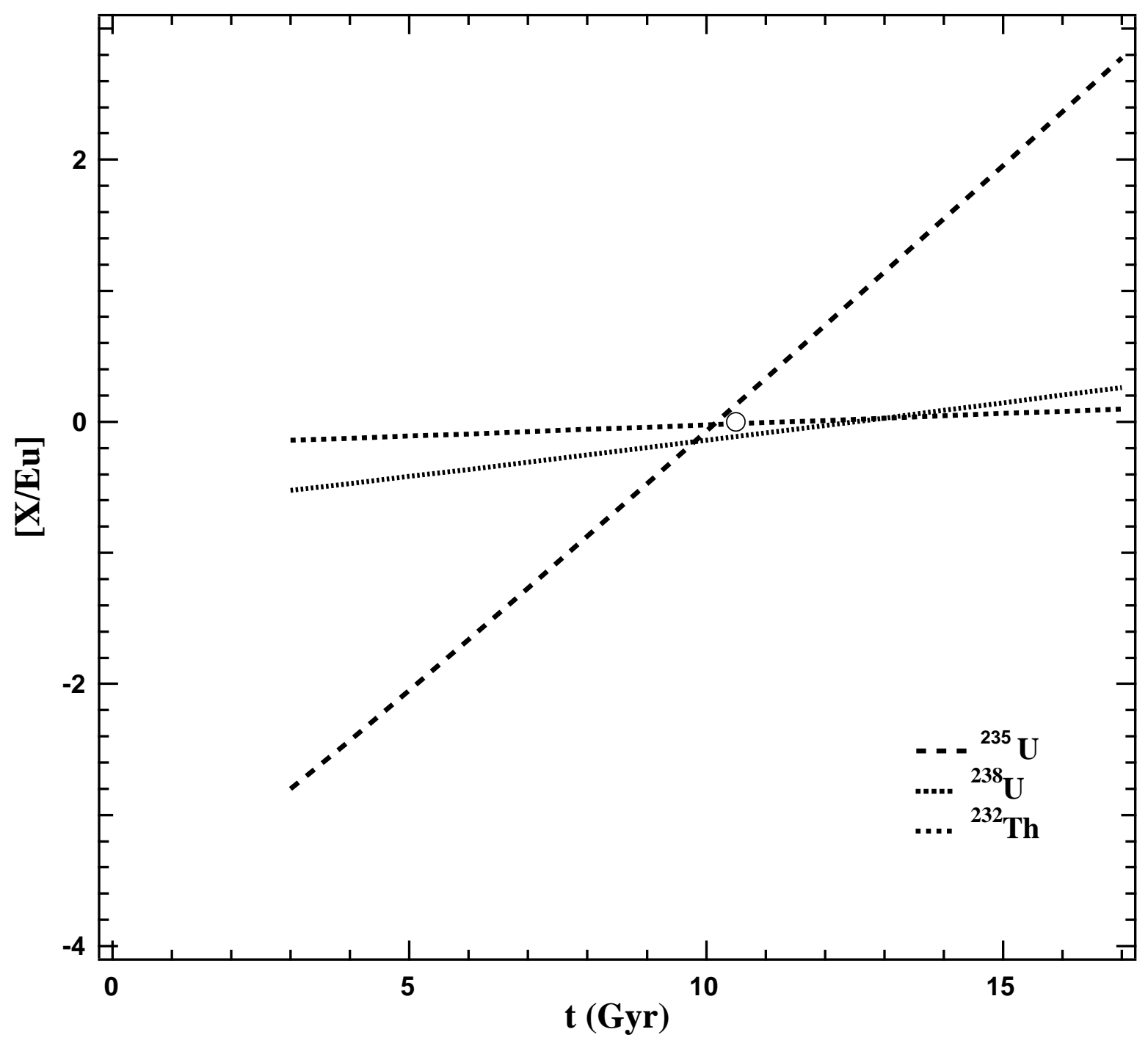




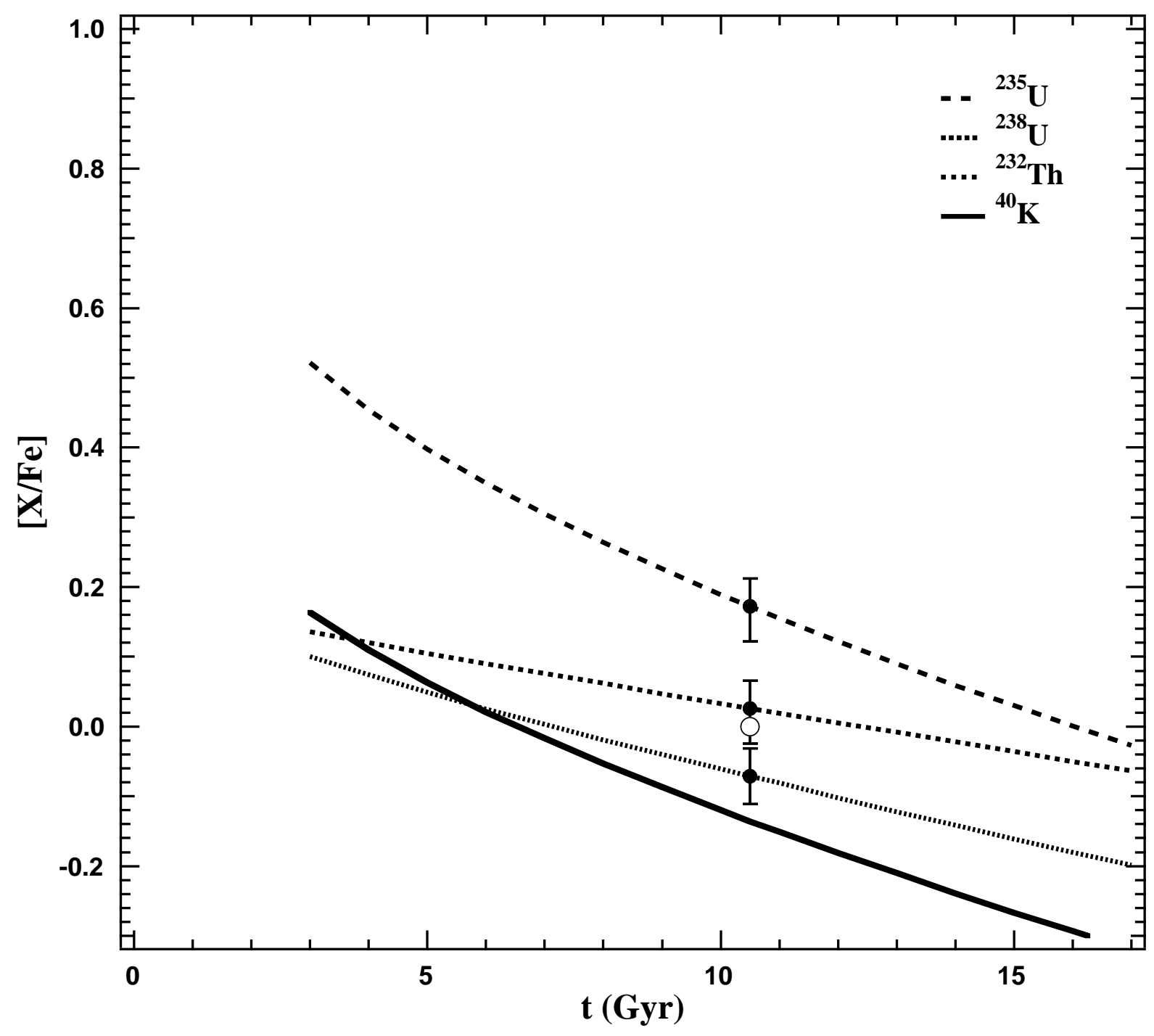




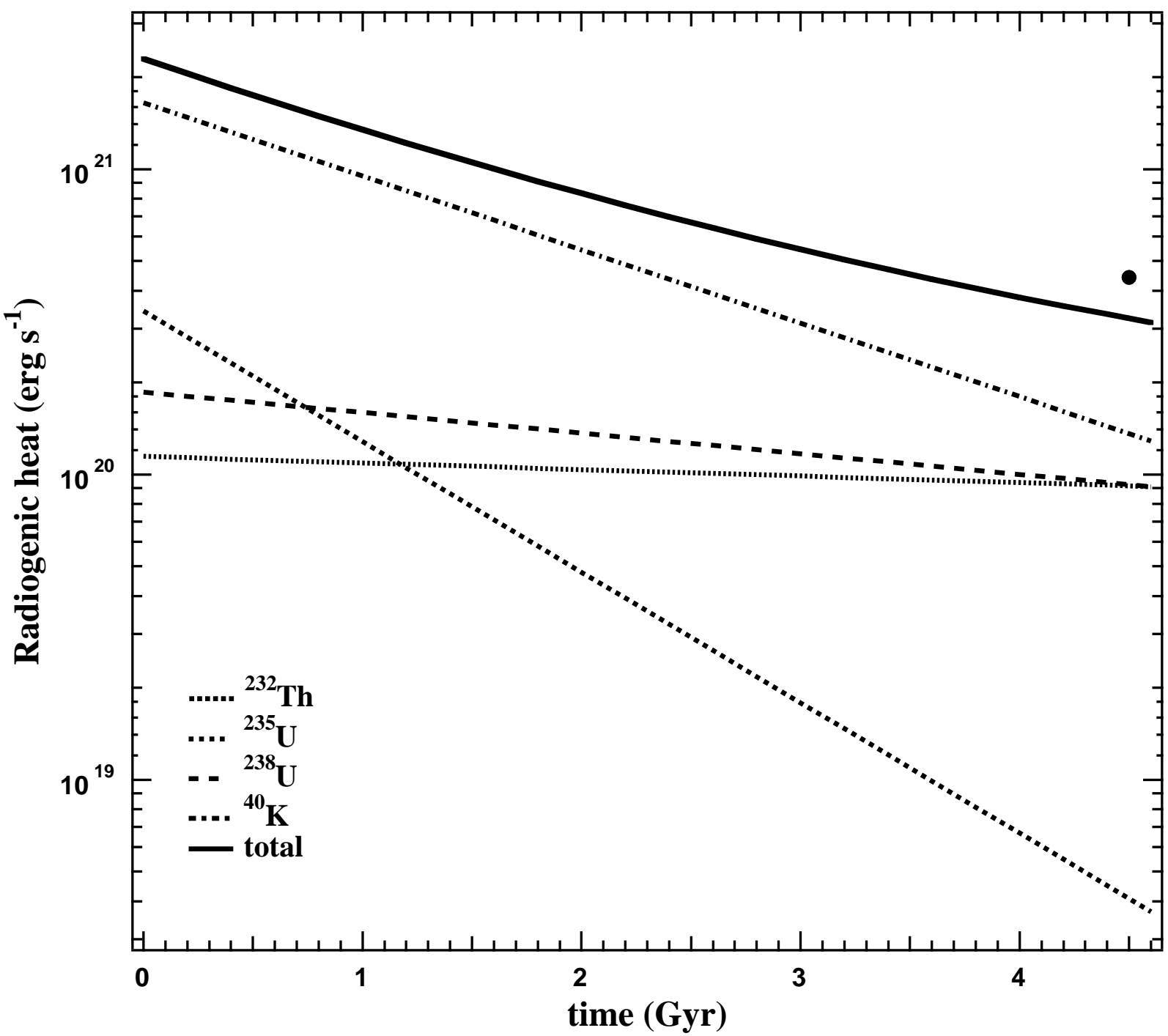




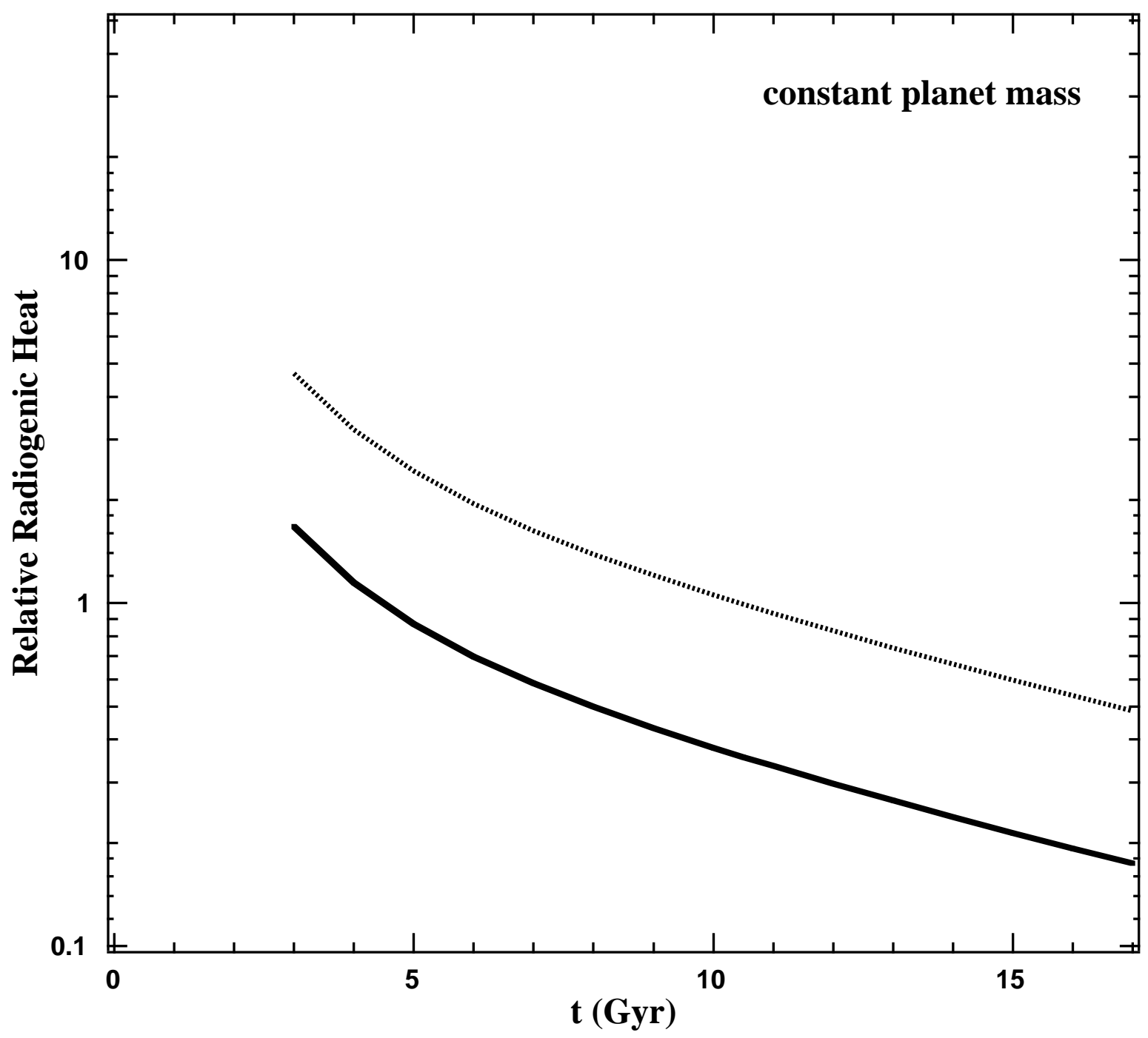




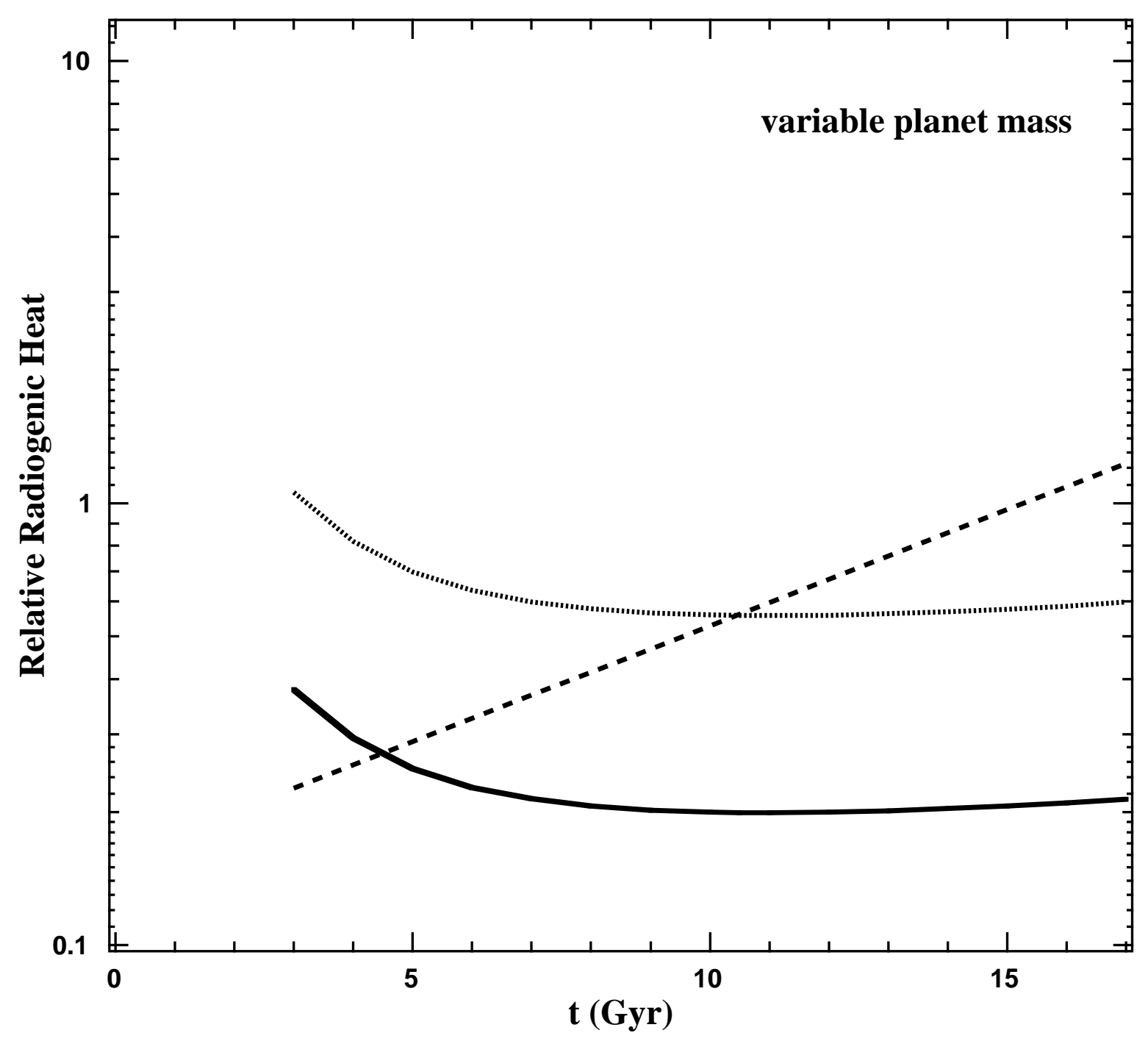

